\title{
Random Young Towers and Quenched Limit Laws
}

\author{
Yaofeng $\mathrm{Su}^{*}$
}

January 22, 2020

\begin{abstract}
We obtain quenched almost sure invariance principle (with convergence rate) for random Young tower. Applications are some i.i.d. perturbations of some non-uniformly expanding maps. In particular, we answer one open question in [BBMD02].
\end{abstract}

\section{Contents}

1 Introduction 1

2 Definitions, Main Theorem and Conventions 3

3 Revisit Random Young Tower $\quad 8$

4 Several Lemmas $\quad 12$

5 Proof of Theorem $2.6 \quad 16$

6 Project From Tower 30

7 Applications $\quad 35$

\section{Introduction}

A collection $\left(\Omega, \mathbb{P}, \sigma,\left(\Delta_{\omega}\right)_{\omega \in \Omega},\left(\mu_{\omega}\right)_{\omega \in \Omega},\left(F_{\omega}\right)_{\omega \in \Omega}\right)$ is called a random dynamical system $(\operatorname{RDS})$ if $(\Omega, \mathbb{P})$ is a probability space, $\sigma: \Omega \rightarrow \Omega$ is an invertible $\mathbb{P}$-preserving

${ }^{*}$ Department of Mathematics, University of Houston, Houston, TX 77204-3008, USA. yfsu@math.uh.edu 
transformation. For each $\omega \in \Omega$, the probability space $\left(\Delta_{\omega}, \mu_{\omega}\right)$ is called a fiber at $\omega . \quad F_{\omega}: \Delta_{\omega} \rightarrow \Delta_{\sigma \omega}$ is called a fiber map, satisfying $\left(F_{\omega}\right)_{*} \mu_{\omega}=\mu_{\sigma \omega} .\left(\mu_{\omega}\right)_{\omega \in \Omega}$ are called equivariant probability measures.

A decreasing series $\rho_{n} \searrow 0$ is called an almost surely mixing rate for the RDS if for a.e. $\omega \in \Omega$, there is a constant $C_{\omega}>0$ and a Banach space $B_{\omega} \subset L^{1}\left(\Delta_{\omega}, \mu_{\omega}\right)$ s.t. for any $n \in \mathbb{N}, \phi_{\omega} \in B_{\omega}, \Psi_{\sigma^{n} \omega} \in L^{\infty}\left(\Delta_{\sigma^{n} \omega}, \mu_{\sigma^{n} \omega}\right)$, there is $C_{\phi, \Psi}>0$ and

$$
\left|\int \phi_{\omega} \cdot \Psi_{\sigma^{n} \omega} \circ F_{\omega}^{n} d \mu_{\omega}-\int \phi_{\omega} d \mu_{\omega} \int \Psi_{\sigma^{n} \omega} d \mu_{\sigma^{n} \omega}\right| \leq C_{\phi, \Psi} \cdot C_{\omega} \cdot \rho_{n} \rightarrow 0,
$$

where $\phi(\omega, \cdot):=\phi_{\omega}(\cdot), \Psi(\omega, \cdot):=\Psi_{\omega}(\cdot)$.

We call the RDS has an uniform almost surely mixing rate if $\operatorname{ess}_{\sup } \operatorname{su}_{\omega \in \Omega} C_{\omega}<\infty$, the RDS has a non-uniform almost surely mixing rate if $\operatorname{ess}_{\sup } \operatorname{up}_{\omega \in \Omega} C_{\omega}=\infty$.

A random Young tower (RYT) is a powerful tool to study almost surely mixing rate for RDS with weak hyperbolicity. The original one is constructed by Baladi, Benedicks and Maume-Deschamps [BBMD02] to obtain the almost surely mixing rate for i.i.d. translations of unimodal maps. In recent years, RYT has been extended and used intensively: Du [Du15] extends [BBMD02] to a more general RYT and applies it to i.i.d. perturbations of a wider class of unimodal maps. Bahsoun, Bose, Li, Ruziboev and Vilarinho [LV18,BBR19] use RYT to obtain almost surely mixing rates for i.i.d. perturbations of some non-uniformly expanding maps.

In [BBMD02], Baladi, Benedicks and Maume-Deschamps ask: if an almost surely mixing rate exists, does quenched central limit theorem (QCLT) hold for the RDS? Furthermore, we can ask: quenched almost surely invariance principle (QASIP), which is a very strong form of quenched limit law strengthening QCLT, quenched functional central limit theorem (QFCLT) and quenched law of iterated logarithm (QLIL), holds for the RDS?

For a RDS with an uniform almost surely mixing rate, there is already extensive literature studying quenched limit laws, see [ALS09, HL18, HS18, DFGTV18, Su19a, Su19b]. However, it is quite natural and more often to see a RDS (e.g. RYT) has a non-uniform almost surely mixing rate. To the best of our knowledge, for such RDS, two papers [AA16, Kif98] do make progress: Abdelkader and Aimino [AA16] study RDS with expanding in average. Inspired by [ALS09], they fix one reference measure, instead of finding equivariant probability measures, to study QCLT. But they finally find an example which fails to have QCLT no matter how fast its almost surely mixing rate is. The correct approach is due to Kifer [Kif98]: assuming equivariant probability measures for RDS. His method is introducing hitting time on $(\Omega, \mathbb{P}, \sigma)$ to induce a new RDS which has an uniform almost surely mixing rate. Then he places several conditions on the hitting time so that the QCLT of the induced RDS can be transferred to the original RDS. However, his conditions are quite complicated and hard to verify. In addition, as he remarks in Proposition 2.2 and Remark 6.5 
in [Kif98], his method has to work on specific cases with an explicit representation. Even in his applications under the assumption that $(\Omega, \sigma)$ is Bernoulli, it is still unclear whether the RDS in [Kif98] has QCLT or not.

In this paper we will not adopt Kifer's method. We will claim clearly that the RYT has QASIP and answer one open question in [BBMD02]. Our approach to QASIP is the random version of martingale approximation similar to [Liv96]. Technical lemmas will be given to overcome the difficulty caused by the unbounded $C_{\omega}$ and obtain the convergence rate of QASIP. This convergence rate is $O\left(n^{\frac{1}{4}+\epsilon}\right)$ when the RDS has almost surely (stretch) exponential mixing rate.

Outline of this paper is as follows: in Section 2 we give the definition of a general RYT in [Du15] and our main theorem for QASIP. In section 3 we revisit RYT in [Du15] and modify/improve some inequalities such that they can be used to prove QASIP. In section 4 several technical lemmas are given. In section 5 we prove our main theorem. In section 6 we project QASIP from RYT down to the RDS with induced Markov structure via semiconjugacies. In section 7, Applications, we apply our result to some i.i.d. perturbations of some non-uniformly expanding maps.

\section{Definitions, Main Theorem and Conventions}

Definition 2.1 (Random Young Tower, see [Du15])

We construct a random Young tower $(\Delta, F)$ in the following steps:

1. Fix a probability space $(\Lambda, \mathcal{B}, m)$, a Bernoulli scheme $(\Omega, \mathbb{P}, \sigma):=\left(S^{\mathbb{Z}}, \nu^{\mathbb{Z}}, \sigma\right)$ where $\nu$ is probability on the measurable space $S$ and $\sigma$ is invertible left shift on $S^{\mathbb{Z}}$.

2. Assume for a.e. $\omega \in \Omega$, there is a countable partition $\mathcal{P}_{\omega}$ of a full measure subset $\mathcal{D}_{\omega}$ of $\Lambda$ and a function $R_{\omega}: \Lambda \rightarrow \mathbb{N}$ such that $R_{\omega}$ is constant on each $U_{\omega} \in \mathcal{P}_{\omega}$.

3. Assume $R_{\omega}(x)$ is a stopping time: if $R_{\omega}(x)=n$ and $\omega_{i}=\omega_{i}^{\prime}$ for $0 \leq i<n$, then $R_{\omega^{\prime}}(x)=n$.

4. For a.e. $\omega \in \Omega, l \in \mathbb{N}$, define $\Delta_{\omega, 0}:=\Lambda$ and the l-th level by

$$
\Delta_{\omega, l}:=\left\{(x, l): x \in \Lambda, R_{\sigma^{-l} \omega}(x)>l\right\} .
$$

Also define a tower at $\omega$ by

$$
\Delta_{\omega}:=\bigcup_{l \geq 0} \Delta_{\omega, l} .
$$


$\Delta_{\omega}$ is endowed with a measure $m_{\omega}$, a $\sigma$-algebra $\mathcal{B}_{\omega}$ and a partition $\mathcal{Z}_{\omega}$ naturally from the probability space $(\Lambda, \mathcal{B}, m)$ and the partitions $\left(\mathcal{P}_{\sigma^{-l}}\right)_{l \geq 0}$.

5. Assume for a.e. $\omega \in \Omega$, there is $F_{\omega}: \Delta_{\omega} \rightarrow \Delta_{\sigma \omega}$ satisfying: if $R_{\sigma^{-l} \omega}(x)>l+1$, then $F_{\omega}(x, l)=(x, l+1)$. If $R_{\sigma^{-l} \omega}(x)=l+1$ and $x \in U_{\sigma^{-l} \omega} \in \mathcal{P}_{\sigma^{-l} \omega}$, then $F_{\omega}$ maps $U_{\sigma^{-l} \omega} \times\{l\}$ bijectively onto $\Delta_{\sigma \omega, 0}$.

6. Define $F_{\omega}^{n}:=F_{\sigma^{n-1} \omega} \circ F_{\sigma^{n-2} \omega} \circ \cdots \circ F_{\sigma \omega} \circ F_{\omega}$, assume the partition $\mathcal{Z}_{\omega}$ is generating for $F_{\omega}$ in the sense that the diameter of the partition $\bigvee_{j=0}^{n}\left(F_{\omega}^{j}\right)^{-1} \mathcal{Z}_{\sigma^{j} \omega}$ tends to 0 as $n \rightarrow \infty$.

7. Assume for a.e. $\omega \in \Omega, m_{\omega}\left(\Delta_{\omega}\right)<\infty$.

8. Assume there is an integer $M \in \mathbb{N},\left\{\epsilon_{i}>0, i=1, \cdots, M\right\}$ and $\left\{t_{i} \in \mathbb{N}, i=\right.$ $1, \cdots, M\}$ with $\operatorname{gcd}\left(t_{i}\right)=1$ such that for a.e. $\omega \in \Omega$, all $1 \leq i \leq M$,

$$
m\left(x \in \Lambda: R_{\omega}(x)=t_{i}\right)>\epsilon_{i} .
$$

9. Extend $R_{\omega}$ to $\Delta_{\omega}$ (still denoted by $R_{\omega}$ ): for any $(x, l) \in \Delta_{\omega}$,

$$
R_{\omega}(x, l):=R_{\sigma^{-l} \omega}(x)-l .
$$

Define $n$-th return time on $\Delta_{\omega}$ inductively: for any $x \in \Delta_{\omega}$,

$$
R_{\omega}^{0}(x):=0, R_{\omega}^{1}(x):=R_{\omega}(x), R_{\omega}^{n}(x):=R_{\omega}^{n-1}(x)+R_{\sigma_{\omega}^{R^{n-1}(x)} \omega}\left(F_{\omega}^{R_{\omega}^{n-1}}(x)\right) .
$$

Define separation time $s_{\omega}: \Delta_{\omega} \times \Delta_{\omega} \rightarrow \mathbb{N} \cup\{\infty\}$ by: $s_{\omega}(x, y)=\inf \left\{n: F_{\omega}^{R_{\omega}^{n}(x)}(x), F_{\omega}^{R_{\omega}^{n}(y)}(y)\right.$ lie in different elements of $\left.\mathcal{Z}_{\sigma^{R_{\omega}^{n}(x)} \omega}\right\}$.

Assume there is a constant $C_{F}>0$ and $\beta \in(0,1)$ such that for a.e. $\omega \in \Omega$ and each element $J_{\omega} \in \mathcal{Z}_{\omega}$, the map $\left.F_{\omega}^{R_{\omega}}\right|_{J_{\omega}}$ and its inverse are non-singular w.r.t. $m$, and for each $x, y \in J_{\omega}$,

$$
\left|\frac{J F_{\omega}^{R_{\omega}}(x)}{J F_{\omega}^{R_{\omega}}(y)}-1\right| \leq C_{F} \cdot \beta^{\left.s_{\sigma} R_{\omega}(x)\right)_{\omega}}\left(F_{\omega}^{R_{\omega}}(x), F_{\omega}^{R_{\omega}}(y)\right) .
$$

10. Assume there is a constant $C>0$ s.t.

$$
\int m\left(x \in \Lambda: R_{\omega}(x)>n\right) d \mathbb{P} \leq C \cdot \rho_{n},
$$

where $\rho_{n}:=e^{-a \cdot n^{b}}$ or $\frac{1}{n^{D}}$ for some constants $a>0, b \in(0,1], D>4$. 
11. Define the random Young tower $(\Delta, F)$ by

$$
\Delta:=\bigcup_{\omega \in \Omega}\{\omega\} \times \Delta_{\omega}, F(\omega, x):=\left(\sigma \omega, F_{\omega} x\right) .
$$

Remark 2.2 For the 3D picture of the RYT's dynamic, see Figure 1 in [BBR19].

\section{Definition 2.3 (Dynamical Lipschitz Cone)}

$\mathcal{F}_{\beta}^{+}:=\left\{\phi: \Delta \rightarrow \mathbb{C} \mid\right.$ there is $C_{\phi}>0$, for any $J_{\omega} \in \mathcal{Z}_{\omega}$, either $\left.\phi_{\omega}\right|_{J_{\omega}}=0$ or

$$
\left.\left.\phi_{\omega}\right|_{J_{\omega}}>0 \text { and for any } x, y \in J_{\omega},\left|\log \frac{\phi_{\omega}(x)}{\phi_{\omega}(y)}\right| \leq C_{\phi} \cdot \beta^{s_{\omega}(x, y)}\right\}
$$

where $C_{\phi}$ is called Lipschitz constant for $\phi$.

\section{Definition 2.4 (Bounded Random Lipschitz Function)}

For any $p \in(1, \infty]$, define:

$$
\begin{gathered}
\mathcal{F}_{\beta, p}^{\mathcal{K}}:=\left\{\phi: \Delta \rightarrow \mathbb{C} \mid \text { there are constants } C_{\phi}>0, \mathcal{K}_{\omega} \geq 1\right. \text { such that } \\
\left.\mathcal{K}_{(\cdot)} \in L^{p}(\Omega),\left|\phi_{\omega}(x)\right| \leq C_{\phi} \text { and }\left|\phi_{\omega}(x)-\phi_{\omega}(y)\right| \leq C_{\phi} \cdot \mathcal{K}_{\omega} \cdot \beta^{s_{\omega}(x, y)}\right\}
\end{gathered}
$$

where $C_{\phi}$ is also called Lipschitz constant for $\phi$.

Remark 2.5 Instead of defining random bounded function as the Section 2.1.2 of [Du15] does, we only define bounded random Lipschitz function in Definition 2.4 for our main purpose in this paper: quenched limit law.

\section{Theorem 2.6 (QASIP for RYT)}

Assume the RYT in Definition 2.1, then for a.e. $\omega \in \Omega$, there are equivariant probability measures $\left(\mu_{\omega}\right)_{\omega \in \Omega}$ on $\left(\Delta_{\omega}\right)_{\omega \in \Omega}$, that is,

$$
\left(F_{\omega}\right)_{*} \mu_{\omega}=\mu_{\sigma \omega}
$$

Moreover, for any $\phi \in \mathcal{F}_{\beta, p}^{\mathcal{K}}$ with fiberwise mean $0: \int \phi_{\omega} d \mu_{\omega}=0$. Define:

$$
\sigma_{n}^{2}(\omega):=\int\left(\sum_{k \leq n} \phi_{\sigma^{k} \omega} \circ F_{\omega}^{k}\right)^{2} d \mu_{\omega} .
$$

Assume $\rho_{n}$ in Definition 2.1 is $e^{-a \cdot n^{b}}$ or $\frac{1}{n^{D}}$ for some constants $a>0, b \in$ $(0,1], D>2+\frac{4 p}{p-1}$. Then $R Y T(\Delta, F)$ satisfies the following: 
1. There is a constant $\sigma^{2} \geq 0$ s.t. $\lim _{n \rightarrow \infty} \frac{\sigma_{n}^{2}(\omega)}{n}=\sigma^{2}$ a.e. $\omega \in \Omega$.

2. If $\sigma^{2}>0$, we have QASIP: there is $\epsilon_{0} \in\left(0, \frac{1}{4}\right)$ s.t. for a.e. $\omega \in \Omega$, there is a Brownian motion $B^{\omega}$ defined on some extension of probability space $\left(\Delta_{\omega}, \mu_{\omega}\right)$, say $\boldsymbol{\Delta}_{\omega}$, such that:

$$
\sum_{k \leq n} \phi_{\sigma^{k} \omega} \circ F_{\omega}^{k}-B_{\sigma_{n}^{2}(\omega)}^{\omega}=O\left(n^{\frac{1}{4}+\epsilon_{0}}\right) \text { a.s. }
$$

the constant indicated in $O(\cdot)$ depends on $\omega$ and $x \in \boldsymbol{\Delta}_{\omega}$, and the term a.s. means almost surely w.r.t. the probability on $\boldsymbol{\Delta}_{\omega}$. Moreover, we have an explicit formula for the convergence rate $O\left(n^{\frac{1}{4}+\epsilon_{0}}\right)$ :

(a) if $\rho_{n}=e^{-a \cdot n^{b}}, \epsilon_{0}>0$ can be chosen to be any small number,

(b) if $\rho_{n}=\frac{1}{n^{D}}, \epsilon_{0}$ can be chosen to be any number between $\left(\epsilon_{D}, \frac{1}{4}\right)$, where

$$
\epsilon_{D}=\max \left\{\frac{1}{4}+\frac{3 \epsilon_{1}-2 \epsilon_{1}^{3}-\epsilon_{1}^{2}}{4}, \epsilon_{1}, \frac{1+\epsilon_{1}}{4}\right\}-\frac{1}{4}
$$

and

$$
\epsilon_{1}=\frac{2 p}{(p-1)(D-2)} \in\left(0, \frac{1}{2}\right)
$$

3. If $\sigma^{2}=0$, we have Coboundary: define $\mu$ on $\Delta$ :

$$
\mu(A):=\int \mu_{\omega}\left(A_{\omega}\right) d \mathbb{P}
$$

where $A$ is measurable on $\Delta$ and $A_{\omega}:=\left\{x \in \Delta_{\omega}:(x, \omega) \in A\right\}$. Then there is measurable function $g$ on $\Delta$ s.t.

$$
\phi_{\sigma \omega} \circ F_{\omega}(x)=g_{\sigma \omega} \circ F_{\omega}(x)-g_{\omega}(x) \text { a.s. }-\mu .
$$

Moreover, if $\rho_{n}=e^{-a \cdot n^{b}}, g \in L^{\infty}(\Delta, \mu)$; if $\rho_{n}=\frac{1}{n^{D}}, g \in L^{\frac{(D-2-\delta) \cdot(p-1)}{(1+\delta) p}}(\Delta, \mu)$ for sufficiently small $\delta>0$.

\section{Remark 2.7}

1. In our section 7, Applications, $p=\infty$.

2. For any $n \geq 1$, define $S_{n}^{\omega}:=\sum_{k \leq n} \phi_{\sigma^{k} \omega} \circ F_{\omega}^{k}$, and piecewise continuous function $S^{n, \omega}$ on $[0,1]$ : 


$$
S_{t}^{n, \omega}:=\frac{S_{i-1}^{\omega}}{\sqrt{n}}+\frac{t-\frac{i-1}{n}}{\frac{i}{n}-\frac{i-1}{n}} \cdot \frac{S_{i}^{\omega}-S_{i-1}^{\omega}}{\sqrt{n}}, t \in\left[\frac{i-1}{n}, \frac{i}{n}\right] \text {, where } 1 \leq i \leq n, \frac{0}{0}:=0 .
$$

Then QASIP for RYT implies QCLT, QLIL, QFCLT for RYT respectively, that is, there is a constant $\sigma^{2}>0$, for a.e. $\omega \in \Omega$,

$$
\begin{gathered}
\frac{S_{n}^{\omega}}{\sqrt{n}} \underset{\mu_{\omega}}{d} N\left(0, \sigma^{2}\right), \\
\limsup _{n \rightarrow \infty} \frac{S_{n}^{\omega}}{\sqrt{n \log \log n}}=\sigma \text { a.s. }-\mu_{\omega}, \\
\liminf _{n \rightarrow \infty} \frac{S_{n}^{\omega}}{\sqrt{n \log \log n}}=-\sigma \text { a.s. }-\mu_{\omega}, \\
S^{n, \omega} \underset{\mu_{\omega}}{\longrightarrow} \sigma \cdot B \text { on } C[0,1],
\end{gathered}
$$

where $B$ is standard one-dimensional Brownian motion.

3. For RYT with polynomially $\rho_{n}=\frac{1}{n^{D}}$, [Du15] obtains mixing rate for $R Y T$ when $D>4$, while [BBR19] obtains mixng rate with a wider range $D>1$, under two more restrictive conditions: $(P 6)$ and $(P 7)$ in [BBR19]. Depending on the RDS we study, restrictive conditions could be added to obtain much stronger results. But in this paper, we just consider the general RYT studied in [Du15], since the restrictive $R Y T$ in [BBR19] in the range $D>4$ is just a special case of the general RYT. We believe QASIP for the restrictive RYT in [BBR19] holds with a little wider range of $D$.

\section{Convention 2.8}

1. $C_{a}$ means a constant $C$ depending on $a$,

2. $\mathbb{E}_{\mu_{\omega}}$ means the expectation w.r.t. $\mu_{\omega}, \mathbb{E}$ means expectation of $\mathbb{P}$.

3. We do not specify the $\sigma$-algebra of a measure space if it could be naturally understood.

4. $a_{n}=O_{a}\left(b_{n}\right)$ means: there is $C_{a}>0$ s.t. $a_{n} \leq C_{a} \cdot b_{n}$ for all $n \in \mathbb{N}$.

5. $a_{n}=C_{a}^{ \pm} \cdot b_{n}$ means: there is $C_{a} \geq 1$ s.t. $C_{a}^{-1} \cdot b_{n} \leq a_{n} \leq C_{a} \cdot b_{n}$. 


\section{Revisit Random Young Tower}

\section{Lemma 3.1 (AC Equivariant Probability and Matching)}

For RYT in Definition 2.1, we have: for a.e. $\omega \in \Omega$, there is an unique absolutely continuous equivariant probability:

$$
\begin{gathered}
d \mu_{\omega}:=h_{\omega} d m_{\omega} \text { on } \Delta_{\omega} \\
h(\omega, \cdot):=h_{\omega}(\cdot) \text { on } \Delta
\end{gathered}
$$

satisfying:

$$
\left(F_{\omega}\right)_{*} \mu_{\omega}=\mu_{\sigma \omega}, h \in \mathcal{F}_{\beta}^{+}, \underset{\omega \in \Omega}{\operatorname{ess} \sup _{\omega}} h_{\omega}<\infty, h_{\omega}>0
$$

$(\Delta, F, \mu)$ is exact, mixing and ergodic.

In addition, there is an integer $l_{0}>0$ such that for any $l \geq l_{0}$, there is $\epsilon_{l} \in(0,1)$, for a.e. $\omega \in \Omega, m_{\omega}\left(\Delta_{\omega, 0} \cap F_{\omega}^{-l} \Delta_{\sigma^{l} \omega, 0}\right)>\epsilon_{l}$.

Furthermore, we have the following matching: let $\lambda_{\omega}, \lambda_{\omega}^{\prime}$ be absolutely continuous probability measures on $\Delta_{\omega}$, with desities: $\frac{d \lambda_{\omega}}{d m_{\omega}}, \frac{d \lambda_{\omega}^{\prime}}{d m_{\omega}} \in \mathcal{F}_{\beta}^{+}$. Recall $R_{\omega}^{i}(x)$ is the $i$-th return time of $(\omega, x) \in \Delta$ into the 0-th level. Define return times on $\bigcup_{\omega \in \Omega}\{\omega\} \times$ $\Delta_{\omega} \times \Delta_{\omega}$ alternatively and recursively:

$$
\begin{aligned}
& \tau_{0}^{\omega}\left(x, x^{\prime}\right):=0, \tau_{1}^{\omega}\left(x, x^{\prime}\right):=R_{\omega}^{l_{0}}(x), \\
& \tau_{2}^{\omega}\left(x, x^{\prime}\right):=\tau_{1}^{\omega}\left(x, x^{\prime}\right)+R_{\sigma_{1}^{\tau_{1}^{\omega}\left(x, x^{\prime}\right)} \omega}^{l_{0}}\left(F_{\omega}^{\tau_{1}^{\omega}\left(x, x^{\prime}\right)} x^{\prime}\right) \\
& \tau_{3}^{\omega}\left(x, x^{\prime}\right):=\tau_{2}^{\omega}\left(x, x^{\prime}\right)+R_{\sigma_{2}^{\tau_{2}^{\omega}\left(x, x^{\prime}\right)}{ }_{\omega}}^{l_{0}}\left(F_{\omega}^{\tau_{2}^{\omega}\left(x, x^{\prime}\right)} x\right), \\
& \tau_{4}^{\omega}\left(x, x^{\prime}\right):=\tau_{3}^{\omega}\left(x, x^{\prime}\right)+R_{\sigma_{3}^{\tau_{3}\left(x, x^{\prime}\right)}{ }_{\omega}}^{l_{0}}\left(F_{\omega}^{\tau_{3}^{\omega}\left(x, x^{\prime}\right)} x^{\prime}\right) \\
& \tau_{5}^{\omega}\left(x, x^{\prime}\right):=\tau_{4}^{\omega}\left(x, x^{\prime}\right)+R_{\sigma_{4}^{\tau_{4}^{\omega}\left(x, x^{\prime}\right)} \omega_{\omega}}^{l_{0}}\left(F_{\omega}^{\tau_{4}^{\omega}\left(x, x^{\prime}\right)} x\right), \\
& T^{\omega}\left(x, x^{\prime}\right):=\min \left\{\tau_{i}^{\omega}\left(x, x^{\prime}\right), i \geq 1:\left(F_{\omega} \times F_{\omega}\right)^{\tau_{i}^{\omega}\left(x, x^{\prime}\right)}\left(x, x^{\prime}\right) \in \Delta_{\sigma_{i}^{\tau_{i}^{\omega}\left(x, x^{\prime}\right)} \omega, 0} \times \Delta_{\sigma_{i}^{\tau_{i}^{\omega}\left(x, x^{\prime}\right)} \omega, 0}\right\}, \\
& T_{0}^{\omega}:=0, T_{1}^{\omega}:=T^{\omega}, \\
& T_{n}^{\omega}\left(x, x^{\prime}\right):=T_{n-1}^{\omega}\left(x, x^{\prime}\right)+T^{\sigma_{n-1}^{T_{n}\left(x, x^{\prime}\right)} \omega}\left(\left(F_{\omega} \times F_{\omega}\right)^{T_{n-1}^{\omega}\left(x, x^{\prime}\right)}\left(x, x^{\prime}\right)\right) .
\end{aligned}
$$

Then there is a constant $C>0, r \in(0,1)$, for a.e. $\omega \in \Omega$,

$$
\left|\left(F_{\omega}^{n}\right)_{*} \lambda_{\omega}-\left(F_{\omega}^{n}\right)_{*} \lambda_{\omega}^{\prime}\right| \leq C \cdot \sum_{i=0}^{\infty} r^{i} \cdot\left(\lambda_{\omega} \times \lambda_{\omega}^{\prime}\right)\left(T_{i}^{\omega} \leq n<T_{i+1}^{\omega}\right),
$$


where $C, r$ only depend on $\beta, C_{F}$ and Lipschitz constants of $\frac{d \lambda_{\omega}}{d m_{\omega}}, \frac{d \lambda_{\omega}^{\prime}}{d m_{\omega}}$.

If $\rho_{n}=e^{-a \cdot n^{b}}$ or $\frac{1}{n^{D}}$ where $a>0, b \in(0,1], D>4$, then

$$
\int m_{\omega}^{\otimes 2}\left(\Delta_{\omega} \times \Delta_{\omega}\right) d \mathbb{P}<\infty .
$$

In addition, for any small $\delta>0$, there are $C=C_{\beta, F, \delta}>0$ and sufficiently small $\alpha=\alpha_{\delta}>0$ s.t.

$$
\int m_{\omega}^{\otimes 2}\left(T_{\left\lfloor n^{\alpha}\right\rfloor}^{\omega}>n\right) d \mathbb{P} \leq C \cdot \frac{1}{n^{D-2-\delta}}
$$

Proof For the proof of (3.1), see Theorem 2.2.1, Proposition 2.3.1, 2.3.3-2.3.4 [Du15]. For the proof of (3.2), see Theorem 3.1.1 [Du15].

Since $\rho_{n} \leq e^{-a \cdot n^{b}}$ implies $\rho_{n} \leq \frac{1}{n^{D}}$ for arbitrary large $D$, so we just take care of the latter case, and refer Corollary 7.1.2 [Du15] for the proof of (3.3). This does not hurt our estimates and calculations of QASIP.

Lemma 3.2 Let $\phi \in \mathcal{F}_{\beta, p}^{\mathcal{K}}, \delta>0$ be any small number. Define probability:

$$
d \lambda_{\omega}:=\frac{\phi_{\omega}+\mathcal{K}_{\omega} \cdot C_{\phi}+2 \cdot C_{\phi}}{\int\left(\phi_{\omega}+\mathcal{K}_{\omega} \cdot C_{\phi}+2 \cdot C_{\phi}\right) d \mu_{\omega}} d \mu_{\omega},
$$

where $C_{\phi}$ is Lipschitz constant for $\phi$. Then there is a constant $C=C_{h, F, \beta, \delta}$,

$$
\int\left|\left(F_{\omega}^{n}\right)_{*} \lambda_{\omega}-\left(F_{\omega}^{n}\right)_{*} \mu_{\omega}\right| d \mathbb{P} \leq C \cdot \frac{1}{n^{D-2-\delta}}
$$

Proof First note that: by (3.1), for any $x, y \in \Delta_{\omega}$, recall $\mathcal{K}_{\omega} \geq 1$,

$$
\frac{d \lambda_{\omega}}{d m_{\omega}}(x) \leq \frac{C_{\phi} \cdot\left(3+\mathcal{K}_{\omega}\right)}{C_{\phi} \cdot\left(1+\mathcal{K}_{\omega}\right)} \cdot \operatorname{ess} \sup _{\omega \in \Omega} h_{\omega} \leq \frac{3+\mathcal{K}_{\omega}}{1+\mathcal{K}_{\omega}} \cdot C_{h} \leq 2 C_{h},
$$

where $C_{h}$ is Lipschitz constant of $h$.

$$
\left|\log \frac{\frac{d \lambda_{\omega}}{d m_{\omega}}(x)}{\frac{d \lambda_{\omega}}{d m_{\omega}}(y)}\right| \leq\left|\log \frac{h_{\omega}(x)}{h_{\omega}(y)}\right|+\left|\log \frac{\phi_{\omega}(x)+\mathcal{K}_{\omega} \cdot C_{\phi}+2 \cdot C_{\phi}}{\phi_{\omega}(y)+\mathcal{K}_{\omega} \cdot C_{\phi}+2 \cdot C_{\phi}}\right| .
$$

Using inequality $\log x \leq x-1$ when $x \geq 1$, the above inequality becomes:

$$
\begin{gathered}
\leq C_{h} \cdot \beta^{s_{\omega}(x, y)}+\frac{\left|\phi_{\omega}(x)-\phi_{\omega}(y)\right|}{\phi_{\omega}(y)+\mathcal{K}_{\omega} \cdot C_{\phi}+2 \cdot C_{\phi}} \\
\leq C_{h} \cdot \beta^{s_{\omega}(x, y)}+\frac{C_{\phi} \cdot \mathcal{K}_{\omega} \cdot \beta^{s_{\omega}(x, y)}}{\phi_{\omega}(y)+\mathcal{K}_{\omega} \cdot C_{\phi}+2 \cdot C_{\phi}} \leq\left(C_{h}+1\right) \cdot \beta^{s_{\omega}(x, y)} .
\end{gathered}
$$


Therefore, $\frac{d \lambda_{\omega}}{d m_{\omega}} \in \mathcal{F}_{\beta}^{+}$with Lipschitz constant $2 C_{h}+1$.

By (3.2), there is a constant $C=C_{\beta, F, h}>0$ and $\alpha=\alpha_{\delta}$ such that

$$
\begin{gathered}
\int\left|\left(F_{\omega}^{n}\right)_{*} \lambda_{\omega}-\left(F_{\omega}^{n}\right)_{*} \mu_{\omega}\right| d \mathbb{P} \leq C \cdot \int \sum_{i=0}^{\infty} r^{i} \cdot\left(\lambda_{\omega} \times \mu_{\omega}\right)\left(T_{i}^{\omega} \leq n<T_{i+1}^{\omega}\right) d \mathbb{P} \\
=C \cdot \int \sum_{i=\left\lfloor n^{\alpha}\right\rfloor}^{\infty} r^{i} \cdot\left(\lambda_{\omega} \times \mu_{\omega}\right)\left(T_{i}^{\omega} \leq n<T_{i+1}^{\omega}\right) d \mathbb{P} \\
+C \cdot \int \sum_{i=0}^{\left\lfloor n^{\alpha}\right\rfloor-1} r^{i} \cdot\left(\lambda_{\omega} \times \mu_{\omega}\right)\left(T_{i}^{\omega} \leq n<T_{i+1}^{\omega}\right) d \mathbb{P} .
\end{gathered}
$$

Note that $r<1$, by (3.5), the above inequality becomes:

$$
\leq C \cdot r^{\left\lfloor n^{\alpha}\right\rfloor}+2 \cdot C_{h}^{2} \cdot C \cdot \int\left(m_{\omega} \times m_{\omega}\right)\left(T_{\left\lfloor n^{\alpha}\right\rfloor}^{\omega}>n\right) d \mathbb{P} .
$$

By (3.2), the above inequality becomes:

$$
\leq C \cdot r^{\left\lfloor n^{\alpha}\right\rfloor}+2 \cdot C_{h}^{2} \cdot C \cdot \frac{1}{n^{D-2-\delta}} \leq C \cdot \frac{1}{n^{D-2-\delta}},
$$

where the last constant $C$ depends on $\alpha_{\delta}, \delta, \beta, F, h$.

\section{Definition 3.3 (Dual Operator)}

$P_{\omega}: L^{1}\left(\Delta_{\omega}, \mu_{\omega}\right) \rightarrow L^{1}\left(\Delta_{\sigma \omega}, \mu_{\sigma \omega}\right)$ is called dual operator for $F_{\omega}: \Delta_{\omega} \rightarrow \Delta_{\sigma \omega}$ if it satisfies: for any $\Psi_{\omega} \in L^{1}\left(\Delta_{\omega}, \mu_{\omega}\right), \Upsilon_{\sigma \omega} \in L^{\infty}\left(\Delta_{\sigma \omega}, \mu_{\sigma \omega}\right)$,

$$
\int \Psi_{\omega} \cdot \Upsilon_{\sigma \omega} \circ F_{\omega} d \mu_{\omega}=\int P_{\omega}\left(\Psi_{\omega}\right) \cdot \Upsilon_{\sigma \omega} d \mu_{\sigma \omega}
$$

\section{Lemma 3.4 (Property of Dual Operator)}

For $R Y T$, the dual operator $P_{\omega}$ for $F_{\omega}$ exists for a.e. $\omega \in \Omega$. Moreover, for a.e. $\omega \in \Omega$, any $i, k \geq 0$, any measurable functions $\Psi, \Upsilon$ on $\Delta$ :

if $\Psi \in L^{\infty}(\Delta, \mu)$,

$$
\left\|P_{\omega} \Psi_{\omega}\right\|_{L^{\infty}\left(\mu_{\sigma \omega}\right)} \leq\left\|\Psi_{\omega}\right\|_{L^{\infty}\left(\mu_{\omega}\right)},
$$

if $\Psi \in L^{1}(\Delta, \mu)$,

$$
\begin{gathered}
\mathbb{E}_{\mu_{\omega}}\left[\Psi_{\sigma^{i} \omega} \circ F_{\omega}^{i} \mid\left(F_{\omega}^{i+1}\right)^{-1} \mathcal{B}_{\sigma^{i+1} \omega}\right]=\left[P_{\sigma^{i} \omega}\left(\Psi_{\sigma^{i} \omega}\right)\right] \circ F_{\omega}^{i+1} \text { in } L^{1}\left(\mu_{\omega}\right), \\
\frac{\left(F_{\omega}^{i}\right)_{*}\left(\Psi_{\omega} d \mu_{\omega}\right)}{d \mu_{\sigma^{i} \omega}}=P_{\omega}^{i}\left(\Psi_{\omega}\right) \text { in } L^{1}\left(\mu_{\sigma \omega}\right)
\end{gathered}
$$


if $\Psi, \Upsilon \in L^{2}(\Delta, \mu)$,

$$
P_{\omega}^{i+k}\left(\Psi_{\sigma^{i} \omega} \circ F_{\omega}^{i} \cdot \Upsilon_{\omega}\right)=P_{\sigma^{i} \omega}^{k}\left(\Psi_{\sigma^{i} \omega} \cdot P_{\omega}^{i}\left(\Upsilon_{\omega}\right)\right) \text { in } L^{1}\left(\mu_{\sigma^{i+k}}\right),
$$

where $P_{\omega}^{i}:=P_{\sigma^{i-1} \omega} \circ \cdots \circ P_{\sigma \omega} \circ P_{\omega}$.

Proof By (3.1), $h_{\omega}>0$ a.e. $\omega \in \Omega$. Similar to Ruelle-Perron-Frobenius operator, it is straightforward to verify (3.6-3.9) via Definition 3.3 and the existence of $P_{\omega}$ : for a.e. $\omega \in \Omega$ :

$$
\left(P_{\omega} \Psi_{\omega}\right)(x)=\frac{1}{h_{\sigma \omega}(x)} \sum_{F_{\omega}(y)=x} \frac{\Psi_{\omega}(y) \cdot h_{\omega}(y)}{J F_{\omega}(y)} \text { in } L^{1}\left(\mu_{\sigma \omega}\right),
$$

where $J$ is Jacobian of $F_{\omega}$ w.r.t. $m$.

\section{Lemma 3.5 (Average Decay)}

For $R Y T$, any $\phi \in \mathcal{F}_{\beta, p}^{\mathcal{K}}$ and any small $\delta>0$, there is $C=C_{\phi} \cdot C_{h, F, \beta, \delta, p} \cdot\|\mathcal{K}\|_{L^{p}}$ such that

$$
\mathbb{E} \int\left|P_{\omega}^{n}\left(\phi_{\omega}-\int \phi_{\omega} d \mu_{\omega}\right)\right| d \mu_{\sigma^{n} \omega} \leq C \cdot \frac{1}{n^{(D-2-\delta) \cdot \frac{p-1}{p}}} .
$$

\section{Proof}

Let

$$
d \lambda_{\omega}:=\frac{\phi_{\omega}+\mathcal{K}_{\omega} \cdot C_{\phi}+2 \cdot C_{\phi}}{\int\left(\phi_{\omega}+\mathcal{K}_{\omega} \cdot C_{\phi}+2 \cdot C_{\phi}\right) d \mu_{\omega}} d \mu_{\omega}
$$

by (3.8):

$\mathbb{E} \int\left|P_{\omega}^{n}\left(\phi_{\omega}-\int \phi_{\omega} d \mu_{\omega}\right)\right| d \mu_{\sigma^{n} \omega}=\int\left|\int\left(\phi_{\omega}+2 C_{\phi}+C_{\phi} \mathcal{K}_{\omega}\right) d \mu_{\omega}\right| \cdot\left|\left(F_{\omega}^{n}\right)_{*} \lambda_{\omega}-\left(F_{\omega}^{n}\right)_{*} \mu_{\omega}\right| d \mathbb{P}$

$$
\leq 3 C_{\phi} \cdot \int\left|\left(F_{\omega}^{n}\right)_{*} \lambda_{\omega}-\left(F_{\omega}^{n}\right)_{*} \mu_{\omega}\right| d \mathbb{P}+C_{\phi} \cdot \int \mathcal{K}_{\omega} \cdot\left|\left(F_{\omega}^{n}\right)_{*} \lambda_{\omega}-\left(F_{\omega}^{n}\right)_{*} \mu_{\omega}\right| d \mathbb{P} .
$$

Using Hölder inequality, let $\frac{1}{p^{\prime}}=1-\frac{1}{p}$, the above inequality becomes:

$$
\leq 3 C_{\phi} \cdot \int\left|\left(F_{\omega}^{n}\right)_{*} \lambda_{\omega}-\left(F_{\omega}^{n}\right)_{*} \mu_{\omega}\right| d \mathbb{P}+C_{\phi} \cdot|| \mathcal{K} \|_{L^{p}} \cdot\left(\int\left|\left(F_{\omega}^{n}\right)_{*} \lambda_{\omega}-\left(F_{\omega}^{n}\right)_{*} \mu_{\omega}\right|^{p^{\prime}} d \mathbb{P}\right)^{\frac{1}{p^{\prime}}} .
$$

Using the fact that $\left|\left(F_{\omega}^{n}\right)_{*} \lambda_{\omega}-\left(F_{\omega}^{n}\right)_{*} \mu_{\omega}\right| \leq 2$ and (3.4), we have

$$
\leq 3 C_{\phi} \cdot C_{h, F, \beta, \delta} \cdot \frac{1}{n^{D-2-\delta}}+2^{\frac{p^{\prime}-1}{p^{\prime}}} C_{\phi} \cdot\|\mathcal{K}\|_{L^{p}} \cdot\left(\int\left|\left(F_{\omega}^{n}\right)_{*} \lambda_{\omega}-\left(F_{\omega}^{n}\right)_{*} \mu_{\omega}\right| d \mathbb{P}\right)^{\frac{1}{p^{\prime}}}
$$




$$
\begin{gathered}
\leq 3 C_{\phi} \cdot C_{h, F, \beta, \delta} \cdot \frac{1}{n^{D-2-\delta}}+2^{\frac{p^{\prime}-1}{p^{\prime}}} C_{\phi} \cdot\|\mathcal{K}\|_{L^{p}} \cdot C_{h, F, \beta, \delta}^{\frac{1}{p^{\prime}}} \cdot \frac{1}{n^{\frac{D-2-\delta}{p^{\prime}}}} \\
\leq C_{\phi} \cdot C_{h, F, \beta, \delta, p} \cdot\|\mathcal{K}\|_{L^{p}} \cdot \frac{1}{\left(n^{D-2-\delta) \cdot \frac{p-1}{p}}\right.} .
\end{gathered}
$$

\section{Several Lemmas}

Lemma 4.1 If $\Psi \in L^{q}(\Delta, \mu)$ with $q>2$, then for any sufficiently small $\delta \in(0, q-$ $2)$, a.e. $\omega \in \Omega$, we have:

$$
\begin{gathered}
\int\left|\Psi_{\sigma^{n} \omega} \circ F_{\omega}^{n}\right|^{q} d \mu_{\omega}=O_{\omega, q}(n), \\
\int\left|\Psi_{\sigma^{n} \omega} \circ F_{\omega}^{n}\right|^{2} d \mu_{\omega}=O_{\omega, q}\left(n^{\frac{2}{q}}\right), \\
\Psi_{\sigma^{n} \omega} \circ F_{\omega}^{n}(x)=O_{\omega, x, \delta}\left(n^{\frac{2+\delta}{q}}\right) \text { a.s. } x \in \Delta_{\omega} .
\end{gathered}
$$

Proof By ergodic theorem:

$$
\frac{\sum_{i \leq n} \int\left|\Psi_{\sigma^{i} \omega} \circ F_{\omega}^{i}\right|^{q} d \mu_{\omega}}{n} \rightarrow \mathbb{E} \int\left|\Psi_{\omega}\right|^{q} d \mu_{\omega} \text { a.e. } \omega \in \Omega .
$$

So

$$
\int\left|\Psi_{\sigma^{n} \omega} \circ F_{\omega}^{n}\right|^{q} d \mu_{\omega}=O_{\omega, q}(n)
$$

and

$$
\int\left|\Psi_{\sigma^{n} \omega} \circ F_{\omega}^{n}\right|^{2} d \mu_{\omega}=O_{\omega, q}\left(n^{\frac{2}{q}}\right)
$$

Since

$$
\int\left|\frac{\Psi_{\sigma^{n} \omega} \circ F_{\omega}^{n}}{n^{\frac{2+\delta}{q}}}\right|^{q} d \mu_{\omega}=O_{\omega, q}\left(\frac{1}{n^{1+\delta}}\right),
$$

by Borel-Cantelli Lemma:

$$
\Psi_{\sigma^{n} \omega} \circ F_{\omega}^{n}(x)=O_{\omega, x, \delta}\left(n^{\frac{2+\delta}{q}}\right) \text { a.s. } x \in \Delta_{\omega} .
$$




\section{Lemma 4.2 (Martingale Convergence Rate)}

If $\Psi \in L^{q}(\Delta, \mu)$ with $q>2,\left(\Psi_{\sigma^{n} \omega} \circ F_{\omega}^{n}\right)_{n \geq 0}$ is (reverse) martingale difference, then for any sufficiently small $\delta>0$, a.e. $\omega \in \Omega$ :

$$
\begin{gathered}
\left\|\sum_{i \leq n} \Psi_{\sigma^{i} \omega} \circ F_{\omega}^{i}\right\|_{L^{q}\left(\mu_{\omega}\right)}=O_{\omega}\left(n^{\frac{1}{2}}\right), \\
\sum_{i \leq n} \Psi_{\sigma^{i} \omega} \circ F_{\omega}^{i}(x)=O_{x, \omega, q, \delta}\left(n^{\frac{1}{2}+\frac{1+\delta}{q}}\right) \text { a.s. }-\mu_{\omega} .
\end{gathered}
$$

Proof By Burkholder-Davis-Gundy inequality and Minkowski inequality, there is a constant $C_{q}$ s.t.

$$
\left\|\sum_{i \leq n} \Psi_{\sigma^{i} \omega} \circ F_{\omega}^{i}\right\|_{L^{q}\left(\mu_{\omega}\right)} \leq C_{q} \cdot\left\|\left(\sum_{i \leq n} \Psi_{\sigma^{i} \omega}^{2} \circ F_{\omega}^{i}\right)^{\frac{1}{2}}\right\|_{L^{q}\left(\mu_{\omega}\right)} \leq C_{q} \cdot \sqrt{\sum_{i \leq n}\left\|\Psi_{\sigma^{i} \omega}^{2} \circ F_{\omega}^{i}\right\|_{L^{q}\left(\mu_{\omega}\right)}} .
$$

Since $\mathbb{E}|| \Psi_{\omega}^{2} \|_{L^{\frac{q}{2}\left(\mu_{\omega}\right)}} \leq\left(\mathbb{E} \int\left|\Psi_{\omega}\right|^{q} d \mu_{\omega}\right)^{\frac{2}{q}}<\infty$, by ergodic theorem, the above inequality becomes: for a.e. $\omega \in \Omega$,

$$
\left\|\sum_{i \leq n} \Psi_{\sigma^{i} \omega} \circ F_{\omega}^{i}\right\|_{L^{q}\left(\mu_{\omega}\right)} \leq C_{q} \cdot \sqrt{\sum_{i \leq n}\left\|\Psi_{\sigma^{i} \omega}^{2}\right\|_{L^{q}\left(\mu_{\sigma^{i} \omega}\right)}}=O_{\omega, q}\left(n^{\frac{1}{2}}\right) .
$$

Then for any $\delta \in\left(0, \frac{q}{2}-1\right)$,

$$
\int\left|\frac{\sum_{i \leq n} \Psi_{\sigma^{i} \omega} \circ F_{\omega}^{i}}{n^{\frac{1}{2}+\frac{1+\delta}{q}}}\right|{ }^{q} d \mu_{\omega}=O_{\omega, q}\left(\frac{1}{n^{q \cdot\left(\frac{1+\delta}{q}\right)}}\right)=O_{\omega, q}\left(\frac{1}{n^{1+\delta}}\right) .
$$

By Borel-Cantelli Lemma:

$$
\sum_{i \leq n} \Psi_{\sigma^{i} \omega} \circ F_{\omega}^{i}(x)=O_{x, \omega, q, \delta}\left(n^{\frac{1}{2}+\frac{1+\delta}{q}}\right) \text { a.s.- } \mu_{\omega} .
$$

\section{Lemma 4.3 (Average vs Quenched)}

If $\psi \in L^{q}(\Delta, \mu)$ with $q \geq 2$ satisfies

$$
\left\|P_{\omega}^{n} \psi_{\omega}\right\|_{L^{q}(\Delta, \mu)}=O_{q, \psi}\left(\frac{1}{n^{d}}\right) \text { with } d>1,
$$

then for any sufficiently small $\delta>0$, a.e. $\omega \in \Omega$

$$
\begin{gathered}
\left\|\sum_{i \leq n} \psi_{\sigma^{i} \omega} \circ F_{\omega}^{i}\right\|_{L^{q}\left(\mu_{\omega}\right)}=O_{\omega, \psi, q}\left(n^{\frac{1}{2}}\right), \\
\sum_{i \leq n} \psi_{\sigma^{i} \omega} \circ F_{\omega}^{i}(x)=O_{x, \omega, q, \delta, \psi}\left(n^{\frac{1}{2}+\frac{1+\delta}{q}}\right) \text { a.s. }-\mu_{\omega} .
\end{gathered}
$$




\section{Proof Let}

$$
\begin{gathered}
g_{\omega}:=\sum_{i \geq 0} P_{\sigma^{-i} \omega}^{i}\left(\psi_{\sigma^{-i} \omega}\right), \\
\Psi_{\omega}:=\psi_{\sigma \omega} \circ F_{\omega}-g_{\sigma \omega} \circ F_{\omega}+g_{\omega},
\end{gathered}
$$

then

$$
\sum_{1 \leq i \leq n} \psi_{\sigma^{i} \omega} \circ F_{\omega}^{i}=\sum_{1 \leq i \leq n} \Psi_{\sigma^{i-1} \omega} \circ F_{\omega}^{i-1}+g_{\sigma^{n} \omega} \circ F_{\omega}^{n}-g_{\omega}
$$

Since

$$
\|g\|_{L^{q}(\Delta, \mu)} \leq \sum_{i \geq 0}\left\|P_{\sigma^{-i \omega}}^{i}\left(\psi_{\sigma^{-i} \omega}\right)\right\|_{L^{q}(\Delta, \mu)}=O_{q, \psi}\left(\sum_{i \geq 1} \frac{1}{n^{d}}\right)<\infty
$$

then by Lemma 4.1,

$$
\left\|g_{\sigma^{n} \omega} \circ F_{\omega}^{n}\right\|_{L^{q}\left(\mu_{\omega}\right)}=O_{\omega, q}\left(n^{\frac{1}{q}}\right) .
$$

By (3.9), for a.e. $\omega \in \Omega$,

$$
\begin{aligned}
P_{\omega} \Psi_{\omega} & =P_{\omega}\left(\psi_{\sigma \omega} \circ F_{\omega}\right)-P_{\omega}\left(g_{\sigma \omega} \circ F_{\omega}\right)+P_{\omega} g_{\omega}=\psi_{\sigma \omega}-g_{\sigma \omega}+P_{\omega} g_{\omega} \\
& =\psi_{\sigma \omega}-\sum_{i \geq 0} P_{\sigma^{-i} \sigma \omega}^{i}\left(\psi_{\sigma^{-i} \sigma \omega}\right)+\sum_{i \geq 0} P_{\sigma^{-i} \omega}^{i+1}\left(\psi_{\sigma^{-i} \omega}\right)=0
\end{aligned}
$$

then by (3.7),

$$
\mathbb{E}_{\mu_{\omega}}\left(\Psi_{\sigma^{i} \omega} \circ F_{\omega}^{i} \mid\left(F_{\omega}^{i+1}\right)^{-1} \mathcal{B}_{\sigma^{i+1} \omega}\right)=\left[P_{\sigma^{i} \omega}\left(\Psi_{\sigma^{i} \omega}\right)\right] \circ F_{\omega}^{i+1}=0,
$$

that is, $\left(\Psi_{\sigma^{i} \omega} \circ F_{\omega}^{i}\right)_{i \geq 0}$ is reverse martingale difference w.r.t. $\left(\left(F_{\omega}^{i}\right)^{-1} \mathcal{B}_{\sigma^{i} \omega}\right)_{i \geq 0}$.

Then by Lemma 4.2,

$$
\left\|\sum_{i \leq n} \Psi_{\sigma^{i} \omega} \circ F_{\omega}^{i}(x)\right\|_{L^{q}\left(\mu_{\omega}\right)}=O_{\omega}\left(n^{\frac{1}{2}}\right) .
$$

Therefore,

$$
\begin{aligned}
\left\|\sum_{1 \leq i \leq n} \psi_{\sigma^{i} \omega} \circ F_{\omega}^{i}\right\|_{L^{q}\left(\mu_{\omega}\right)} & \leq\left\|\sum_{1 \leq i \leq n} \Psi_{\sigma^{i-1} \omega} \circ F_{\omega}^{i-1}\right\|_{L^{q}\left(\mu_{\omega}\right)}+\left\|g_{\sigma^{n} \omega} \circ F_{\omega}^{n}\right\|_{L^{q}\left(\mu_{\omega}\right)}+\left\|g_{\omega}\right\|_{L^{q}\left(\mu_{\omega}\right)} \\
& =O_{\omega}\left(n^{\frac{1}{2}}\right)+O_{\omega}\left(n^{\frac{1}{q}}\right)+O_{\omega}(1)=O_{\omega}\left(n^{\frac{1}{2}}\right) .
\end{aligned}
$$

Then for any $\delta \in\left(0, \frac{q}{2}-1\right)$,

$$
\int\left|\frac{\sum_{i \leq n} \psi_{\sigma^{i} \omega} \circ F_{\omega}^{i}}{n^{\frac{1}{2}+\frac{1+\delta}{q}}}\right|^{q} d \mu_{\omega}=O_{\omega, q}\left(\frac{1}{n^{q \cdot\left(\frac{1+\delta}{q}\right)}}\right)=O_{\omega, q}\left(\frac{1}{n^{1+\delta}}\right) .
$$


By Borel-Cantelli Lemma:

$$
\sum_{i \leq n} \psi_{\sigma^{i} \omega} \circ F_{\omega}^{i}(x)=O_{x, \omega, q, \delta, \psi}\left(n^{\frac{1}{2}+\frac{1+\delta}{q}}\right) \text { a.s. }-\mu_{\omega} .
$$

\section{Lemma 4.4 (Regularity)}

If $\phi \in \mathcal{F}_{\beta, p}^{\mathcal{K}}$ with Lipschitz constant $C_{\phi}$, then $\left(P_{\omega}^{n} \phi_{\omega}\right)_{\omega \in \Omega} \in \mathcal{F}_{\beta, p}^{\mathcal{K} \circ \sigma^{-n}+C_{h, F}}$ with Lipschitz constant $C_{\phi}$ for any $n \in \mathbb{N}, C_{h, F}:=C_{h}+e^{C_{h}} C_{F}+e^{C_{h}+C_{F}} C_{h}$.

Proof By Lemma 3.4, $\left\|P_{\omega}^{n} \phi_{\omega}\right\|_{L^{\infty}\left(\mu_{\sigma} n_{\omega}\right)}<\infty$. By (3.10), we have

$$
\begin{aligned}
& P_{\omega}^{n} \phi_{\omega}(x)=\frac{1}{h_{\sigma^{n} \omega}(x)} \sum_{F_{\omega}^{n}\left(z_{x}\right)=x} \frac{\phi_{\omega}\left(z_{x}\right) \cdot h_{\omega}\left(z_{x}\right)}{J F_{\omega}^{n}\left(z_{x}\right)}, \\
& P_{\omega}^{n} \phi_{\omega}(y)=\frac{1}{h_{\sigma^{n} \omega}(y)} \sum_{F_{\omega}^{n}\left(z_{y}\right)=y} \frac{\phi_{\omega}\left(z_{y}\right) \cdot h_{\omega}\left(z_{y}\right)}{J F_{\omega}^{n}\left(z_{y}\right)},
\end{aligned}
$$

where $x, y \in \Delta_{\sigma^{n} \omega, l}, z_{x}, z_{y} \in I_{n-l}^{\omega} \in \bigvee_{j=0}^{n-l}\left(F_{\omega}^{j}\right)^{-1} \mathcal{Z}_{\sigma^{j} \omega}, F_{\omega}^{n-l}: I_{n-l}^{\omega} \rightarrow \Delta_{\sigma^{n-l} \omega, 0}$ is bijective. Then

$$
\begin{gathered}
\left|P_{\omega}^{n} \phi_{\omega}(x)-P_{\omega}^{n} \phi_{\omega}(y)\right|=\mid \frac{1}{h_{\sigma^{n} \omega}(x)} \sum_{F_{\omega}^{n}\left(z_{x}\right)=x} \frac{\phi_{\omega}\left(z_{x}\right) \cdot h_{\omega}\left(z_{x}\right)}{J F_{\omega}^{n}\left(z_{x}\right)} \\
-\frac{1}{h_{\sigma^{n}}(y)} \sum_{F_{\omega}^{n}\left(z_{y}\right)=y} \frac{\phi_{\omega}\left(z_{y}\right) \cdot h_{\omega}\left(z_{y}\right)}{J F_{\omega}^{n}\left(z_{y}\right)}|=| \frac{1}{h_{\sigma^{n} \omega}(x)} \sum_{F_{\omega}^{n}\left(z_{x}\right)=x} \frac{\left(\phi_{\omega}\left(z_{x}\right)-\phi_{\omega}\left(z_{y}\right)\right) \cdot h_{\omega}\left(z_{x}\right)}{J F_{\omega}^{n}\left(z_{x}\right)} \\
+\sum_{F_{\omega}^{n}\left(z_{y}\right)=y} \phi_{\omega}\left(z_{y}\right) \cdot\left(\frac{h_{\omega}\left(z_{x}\right)}{J F_{\omega}^{n}\left(z_{x}\right) \cdot h_{\sigma^{n} \omega}(x)}-\frac{h_{\omega}\left(z_{y}\right)}{J F_{\omega}^{n}\left(z_{y}\right) \cdot h_{\sigma^{n}}(y)}\right) \mid \\
\leq C_{\phi} \cdot \mathcal{K}_{\omega} \cdot \beta^{s_{\sigma} n_{\omega}(x, y)}+C_{\phi} \cdot \sum_{F_{\omega}^{n}\left(z_{y}\right)=y} \frac{h_{\omega}\left(z_{y}\right)}{J F_{\omega}^{n}\left(z_{y}\right) \cdot h_{\sigma^{n}}(y)} \cdot\left|1-\frac{h_{\omega}\left(z_{x}\right)}{\frac{J F_{\omega}^{n}\left(z_{x}\right) \cdot h_{\sigma} n_{\omega}(x)}{h_{\omega}\left(z_{y}\right)}}\right| .
\end{gathered}
$$

Using inequality $\left|1-z_{1} z_{2} z_{3}\right| \leq\left|1-z_{1}\right|+\left|z_{1}\right|\left|1-z_{2}\right|+\left|z_{1}\right|\left|z_{2}\right|\left|1-z_{3}\right|$, we have

$$
\begin{aligned}
& \leq C_{\phi} \cdot\left(\mathcal{K} \circ \sigma^{-n}\right)_{\sigma^{n} \omega} \cdot \beta^{s_{\sigma^{n}}(x, y)}+C_{\phi} \cdot C_{h, F} \cdot \beta^{s_{\sigma^{n} \omega}(x, y)} \\
& \leq C_{\phi} \cdot\left(C_{h, F}+\mathcal{K} \circ \sigma^{-n}\right)_{\sigma^{n} \omega} \cdot \beta^{s_{\sigma} n_{\omega}(x, y)}
\end{aligned}
$$




\section{Proof of Theorem 2.6}

The equivariant probability measures $\left(\mu_{\omega}\right)_{\omega \in \Omega}$ have been obtained in Lemma 3.1. So it remains to prove Coboundary or QASIP and its convergence rate. Recall the conditions in Theorem 2.6:

$$
\begin{gathered}
\phi \in \mathcal{F}_{\beta, p}^{\mathcal{K}} \text { with } \int \phi_{\omega} d \mu_{\omega}=0, \\
\rho_{n}=e^{-a \cdot n^{b}} \text { or } \frac{1}{n^{D}} \text { for some constants } \\
a>0, b \in(0,1], D>2+\frac{4 \cdot p}{p-1} .
\end{gathered}
$$

\section{Martingale Decomposition}

\section{Lemma 5.1 (Decomposition)}

Let

$$
\begin{gathered}
g_{\omega}:=\sum_{i \geq 0} P_{\sigma^{-i} \omega}^{i}\left(\phi_{\sigma^{-i} \omega}\right), \\
g(\omega, \cdot):=g_{\omega}(\cdot), \\
\psi_{\omega}:=\phi_{\sigma \omega} \circ F_{\omega}-g_{\sigma \omega} \circ F_{\omega}+g_{\omega},
\end{gathered}
$$

then for any sufficiently small $\delta>0$ s.t. $\psi, g \in L^{\frac{(D-2-\delta) \cdot(p-1)}{(\delta+1) \cdot p}}(\Delta, \mu) \subseteq L^{4}(\Delta, \mu)$. Besides, for a.e. $\omega \in \Omega$, we have decomposition:

$$
\sum_{1 \leq i \leq n} \phi_{\sigma^{i} \omega} \circ F_{\omega}^{i}=\sum_{1 \leq i \leq n} \psi_{\sigma^{i-1} \omega} \circ F_{\omega}^{i-1}+g_{\sigma^{n} \omega} \circ F_{\omega}^{n}-g_{\omega}
$$

where $\left(\psi_{\sigma^{i} \omega} \circ F_{\omega}^{i}\right)_{i \geq 0}$ is a reverse martingale difference w.r.t. $\left(\left(F_{\omega}^{i}\right)^{-1} \mathcal{B}_{\sigma^{i} \omega}\right)_{i \geq 0}$. Moreover,

1. if $\rho_{n}=e^{-a \cdot n^{b}}, g \in L^{\infty}(\Delta, \mu)$,

2. if $\rho_{n}=\frac{1}{n^{D}}, g \in L^{\frac{(D-2-\delta) \cdot(p-1)}{(1+\delta) \cdot p}}(\Delta, \mu)$ and

$$
\begin{gathered}
\int\left|g_{\sigma^{n} \omega} \circ F_{\omega}^{n}\right|^{2} d \mu_{\omega}=O_{\omega, \delta}\left(n^{\frac{2(1+\delta) p}{(D-2-\delta)(p-1)}}\right) \text { a.e. } \omega \in \Omega, \\
g_{\sigma^{n} \omega} \circ F_{\omega}^{n}(x)=O_{\omega, x, \delta}\left(n^{\frac{(2+\delta)(1+\delta) p}{(D-2-\delta)(p-1)}}\right) \text { a.s. } x \in \Delta_{\omega} .
\end{gathered}
$$


Proof Since $\phi \in L^{\infty}(\Delta, \mu)$, let $q:=\frac{(D-2-\delta) \cdot(p-1)}{(1+\delta) \cdot p}>4$ for sufficiently small $\delta$, by Lemma 3.5 and (3.6),

$$
\begin{gathered}
\|g\|_{L^{q}(\Delta, \mu)} \leq \sum_{i \geq 0}\left\|P_{\sigma^{-i} \omega}^{i}\left(\phi_{\sigma^{-i} \omega}\right)\right\|_{L^{q}(\Delta, \mu)} \\
\leq C_{\phi}+\sum_{i \geq 1}\left[\mathbb{E} \int\left|P_{\sigma^{-i} \omega}^{i}\left(\phi_{\sigma^{-i} \omega}\right)\right| d \mu_{\omega}\right]^{\frac{1}{q}} \cdot C_{\phi}^{\frac{q-1}{q}} \\
\leq C_{\phi}+\sum_{i \geq 1} C_{\phi}^{\frac{q-1}{q}} \cdot\left(C_{\phi} \cdot C_{h, F, \beta, \delta, p} \cdot\|\mathcal{K}\|_{L^{p}}\right)^{\frac{1}{q}} \cdot \frac{1}{i^{1+\delta}}<\infty .
\end{gathered}
$$

Therefore we have: if $\rho_{n}=e^{-a \cdot n^{b}}$, then $\rho_{n} \leq \frac{1}{n^{d}}$ for any $d \gg 4$. So

$$
\begin{gathered}
\|g\|_{L} \frac{(d-2-\delta)(p-1)}{(1+\delta) p}(\Delta, \mu) \\
\leq C_{\phi}+\sum_{i \geq 1} C_{\phi}^{\frac{d-1}{d}} \cdot\left(C_{\phi} \cdot C_{h, F, \beta, \delta, p} \cdot\|\mathcal{K}\|_{L^{p}}\right)^{\frac{1}{d}} \cdot \frac{1}{i^{1+\delta}} \\
\leq C_{\phi}+\sum_{i \geq 1} C_{\phi} \cdot \max \left\{1, C_{\phi} \cdot C_{h, F, \beta, \delta, p} \cdot\|\mathcal{K}\|_{L^{p}}\right\} \cdot \frac{1}{i^{1+\delta}}<\infty
\end{gathered}
$$

Since the right hand side does not depend on $d$, so $g \in L^{\infty}(\Delta, \mu)$; if $\rho_{n}=\frac{1}{n^{D}}$, $g \in L^{q}(\Delta, \mu)$. Then by Lemma 4.1 ,

$$
\begin{gathered}
\int\left|g_{\sigma^{n} \omega} \circ F_{\omega}^{n}\right|^{2} d \mu_{\omega}=O_{\omega, \delta}\left(n^{\frac{2(1+\delta) p}{(D-2-\delta)(p-1)}}\right) \text { a.e. } \omega \in \Omega \\
g_{\sigma^{n} \omega} \circ F_{\omega}^{n}(x)=O_{\omega, x, \delta}\left(n^{\frac{(2+\delta)(1+\delta) p}{(D-2-\delta)(p-1)}}\right) \text { a.s. } x \in \Delta_{\omega} .
\end{gathered}
$$

Since $\phi \in \mathcal{F}_{\beta, p}^{\mathcal{K}}$, so $\psi \in L^{q}(\Delta, \mu)$. By $(3.9)$, for a.e. $\omega \in \Omega$,

$$
\begin{aligned}
P_{\omega} \psi_{\omega} & =P_{\omega}\left(\phi_{\sigma \omega} \circ F_{\omega}\right)-P_{\omega}\left(g_{\sigma \omega} \circ F_{\omega}\right)+P_{\omega} g_{\omega}=\phi_{\sigma \omega}-g_{\sigma \omega}+P_{\omega} g_{\omega} \\
& =\phi_{\sigma \omega}-\sum_{i \geq 0} P_{\sigma^{-i} \sigma \omega}^{i}\left(\phi_{\sigma^{-i} \sigma \omega}\right)+\sum_{i \geq 0} P_{\sigma^{-i} \omega}^{i+1}\left(\phi_{\sigma^{-i} \omega}\right)=0
\end{aligned}
$$

then by (3.7),

$$
\mathbb{E}_{\mu_{\omega}}\left(\psi_{\sigma^{i} \omega} \circ F_{\omega}^{i} \mid\left(F_{\omega}^{i+1}\right)^{-1} \mathcal{B}_{\sigma^{i+1} \omega}\right)=\left[P_{\sigma^{i} \omega}\left(\psi_{\sigma^{i} \omega}\right)\right] \circ F_{\omega}^{i+1}=0
$$

that is, $\left(\psi_{\sigma^{i} \omega} \circ F_{\omega}^{i}\right)_{i \geq 0}$ is reverse martingale difference w.r.t. $\left(\left(F_{\omega}^{i}\right)^{-1} \mathcal{B}_{\sigma^{i} \omega}\right)_{i \geq 0}$. 


\section{Coboundary}

Lemma 5.2 Define $\eta_{n}^{2}(\omega):=\int\left(\sum_{i \leq n} \psi_{\sigma^{i} \omega} \circ F_{\omega}^{i}\right)^{2} d \mu_{\omega}$. Then for sufficiently small $\delta>0$, a.e. $\omega \in \Omega$,

$$
\sigma_{n}^{2}(\omega)-\eta_{n-1}^{2}(\omega)=O_{\omega, \delta}\left(n^{\frac{1}{2}+\frac{(1+\delta) \cdot p}{(D-2-\delta) \cdot(p-1)}}\right) .
$$

Let $\sigma^{2}:=\mathbb{E} \int \psi_{\omega}^{2} d \mu_{\omega}$, then a.e. $\omega \in \Omega$,

$$
\lim _{n \rightarrow \infty} \frac{\sigma_{n}^{2}(\omega)}{n}=\lim _{n \rightarrow \infty} \frac{\eta_{n}^{2}(\omega)}{n}=\sigma^{2}
$$

If $\sigma^{2}=0$, then

$$
\phi \circ F-g \circ F+g=0 \text { a.s. }-\mu,
$$

that is,

$$
\phi_{\sigma \omega} \circ F_{\omega}(x)-g_{\sigma \omega} \circ F_{\omega}(x)+g_{\omega}(x)=0 \text { a.s. }-\mu .
$$

If $\sigma^{2}>0$, there is a constant $C_{\omega} \in[1, \infty)$ s.t. $\eta_{n}^{2}(\omega)=C_{\omega}^{ \pm} \cdot n$.

Proof For sufficiently small $\delta>0$, let $q:=\frac{(D-2-\delta) \cdot(p-1)}{(1+\delta) \cdot p}>4$. Then by (5.1),

$$
\begin{gathered}
\sigma_{n}^{2}(\omega)-\eta_{n-1}^{2}(\omega)=\int\left(\sum_{i \leq n} \phi_{\sigma^{i} \omega} \circ F_{\omega}^{i}\right)^{2} d \mu_{\omega}-\int\left(\sum_{i \leq n} \psi_{\sigma^{i-1} \omega} \circ F_{\omega}^{i-1}\right)^{2} d \mu_{\omega} \\
=\int\left(g_{\sigma^{n} \omega} \circ F_{\omega}^{n}-g_{\omega}\right) \cdot\left(g_{\sigma^{n} \omega} \circ F_{\omega}^{n}-g_{\omega}+2 \sum_{i \leq n} \psi_{\sigma^{i-1} \omega} \circ F_{\omega}^{i-1}\right) d \mu_{\omega}
\end{gathered}
$$

By Lemma 5.1, for a.e. $\omega \in \Omega$,

$$
\begin{gathered}
=\int\left(g_{\sigma^{n} \omega} \circ F_{\omega}^{n}-g_{\omega}\right)^{2} d \mu_{\omega}+2 \int\left(g_{\sigma^{n} \omega} \circ F_{\omega}^{n}-g_{\omega}\right) \cdot\left(\sum_{i \leq n} \psi_{\sigma^{i-1} \omega} \circ F_{\omega}^{i-1}\right) d \mu_{\omega} \\
\leq O_{\omega, \delta}\left(n^{\frac{2}{q}}\right)+O_{\omega, \delta}\left(n^{\frac{1}{q}}\right) \cdot\left\|\sum_{i \leq n} \psi_{\sigma^{i-1} \omega} \circ F_{\omega}^{i-1}\right\|_{L^{2}\left(\mu_{\omega}\right)} \\
\leq O_{\omega, \delta}\left(n^{\frac{2}{q}}\right)+O_{\omega, \delta}\left(n^{\frac{1}{q}+\frac{1}{2}}\right)=O_{\omega, \delta}\left(n^{\frac{1}{q}+\frac{1}{2}}\right) .
\end{gathered}
$$

By (5.2) and ergodic theorem, for a.e. $\omega \in \Omega$,

$$
\lim _{n \rightarrow \infty} \frac{\sigma_{n}^{2}(\omega)}{n}=\lim _{n \rightarrow \infty} \frac{\eta_{n}^{2}(\omega)}{n}=\mathbb{E} \int \psi_{\omega}^{2} d \mu_{\omega}=\sigma^{2} .
$$


Then either $\mathbb{E} \int \psi_{\omega}^{2} d \mu_{\omega}=0$, that is, the Coboundary:

$$
\phi_{\sigma \omega} \circ F_{\omega}(x)-g_{\sigma \omega} \circ F_{\omega}(x)+g_{\omega}(x)=0 \text { a.s. }-\mu,
$$

or $\mathbb{E} \int \psi_{\omega}^{2} d \mu_{\omega}>0$, then there is $C_{\omega} \geq 1$ s.t.

$$
\frac{1}{C_{\omega}} \leq \frac{\eta_{n}^{2}(\omega)}{n} \leq C_{\omega}
$$

\section{Brownian motion Approximation}

From now on, we assume (5.3), i.e. $\mathbb{E} \int \psi_{\omega}^{2} d \mu_{\omega}>0$ :

\section{Lemma 5.3 (Brownian motion Approximation)}

Let $\epsilon \in\left(0, \frac{1}{2}\right), \gamma:=\frac{1}{4 \epsilon}$, define

$$
R_{n}(\omega):=\sum_{i \geq n} \frac{\psi_{\sigma^{i} \omega} \circ F_{\omega}^{i}}{\eta_{i}^{2 \gamma}(\omega)}, \delta_{n}^{2}(\omega):=\int R_{n}^{2}(\omega) d \mu_{\omega},
$$

where $\eta_{n}^{2}(\omega):=\int\left(\sum_{i \leq n} \psi_{\sigma^{i} \omega} \circ F_{\omega}^{i}\right)^{2} d \mu_{\omega}$. Then there is $C_{\omega, \gamma} \geq 1$ s.t.

$$
\delta_{n}^{2}(\omega)=C_{\omega, \gamma}^{ \pm} \cdot \sigma_{n}^{2-4 \gamma}(\omega) \rightarrow 0 .
$$

Since $R_{n}(\omega)$ is $\left(F_{\omega}^{n}\right)^{-1} \mathcal{B}_{\sigma^{n} \omega^{-}}$measurable, so $\left(R_{n}(\omega)\right)_{n \geq 0}$ is reverse martingale w.r.t. $\left(\left(F_{\omega}^{n}\right)^{-1} \mathcal{B}_{\sigma^{n} \omega}\right)_{n \geq 0}$. Therefore, by Theorem 2 in [SH83], there is an extension $\boldsymbol{\Delta}_{\omega}$ of probability space $\left(\Delta_{\omega}, \mu_{\omega}\right)$, Brownian motion $B^{\omega}$ defined on $\left(\boldsymbol{\Delta}_{\omega}, \mathbf{Q}_{\omega}\right)$ and decreasing stopping time $\tau_{i}^{\omega} \searrow 0$, a constant $C_{\omega} \geq 1$ such that

$$
\begin{gathered}
R_{n}(\omega)=B_{\tau_{n}^{\omega}}^{\omega}, \\
\mathbb{E}_{\mathbf{Q}_{\omega}}\left[\tau_{n}^{\omega}-\tau_{n+1}^{\omega} \mid \mathcal{G}_{n+1}^{\omega}\right]=\mathbb{E}_{\mu_{\omega}}\left[\frac{\psi_{n}^{2} \circ F_{\omega}^{n}}{\eta_{n}^{4 \gamma}} \mid\left(F_{\omega}^{n+1}\right)^{-1} \mathcal{B}_{\sigma^{n+1} \omega}\right], \\
\mathbb{E}_{\mu_{\omega}}\left[\frac{\psi_{n}^{2 q} \circ F_{\omega}^{n}}{\eta_{n}^{4 q \gamma}} \mid\left(F_{\omega}^{n+1}\right)^{-1} \mathcal{B}_{\sigma^{n+1} \omega}\right]=C_{\omega}^{ \pm} \cdot \mathbb{E}_{\mathbf{Q}_{\omega}}\left[\left(\tau_{n}^{\omega}-\tau_{n+1}^{\omega}\right)^{q} \mid \mathcal{G}_{n+1}^{\omega}\right],
\end{gathered}
$$

where $q \geq 1$ and $\mathcal{G}_{n}^{\omega}=\sigma\left(\tau_{i}^{\omega},\left(F_{\omega}^{i}\right)^{-1} \mathcal{B}_{\sigma^{i} \omega}, i \geq n\right)$. Moreover, if

$$
\tau_{n}^{\omega}-\delta_{n}^{2}(\omega)=O\left(\delta_{n}^{2+2 \epsilon}(\omega)\right) \text { a.s. },
$$

then

$$
\left|\sum_{i \leq n} \psi_{\sigma^{i} \omega} \circ F_{\omega}^{i}-B_{\eta_{n}^{2}(\omega)}^{\omega}\right|=O\left(n^{\frac{1}{4}+\frac{3 \epsilon-2 \epsilon^{3}-\epsilon^{2}}{4}}\right) \text { a.s. },
$$

where the constants in $O(\cdot)$ depend on $\omega, \epsilon$ and $x \in \boldsymbol{\Delta}_{\omega}$. 
Proof To prove (5.4), first note that: by ergodic theorem,

$\frac{\eta_{n}^{2}(\omega)}{n}=\frac{\int\left(\sum_{i \leq n} \psi_{\sigma^{i} \omega} \circ F_{\omega}^{i}\right)^{2} d \mu_{\omega}}{n}=\frac{\sum_{i \leq n} \int\left(\psi_{\sigma^{i} \omega}\right)^{2} d \mu_{\sigma^{i} \omega}}{n} \rightarrow \mathbb{E} \int \psi_{\omega}^{2} d \mu_{\omega}>0$ a.e. $\omega \in \Omega$,

that is: there is $C_{\omega} \geq 1$ s.t. $\eta_{n}^{2}(\omega)=C_{\omega}^{ \pm} \cdot n, \eta_{n+1}^{2}(\omega)=C_{\omega}^{ \pm} \cdot \eta_{n}^{2}(\omega)$.

Second note that: since $\left(\psi_{\sigma^{i} \omega} \circ F_{\omega}^{i}\right)_{i \geq 1}$ is reverse martingale difference, then

$$
\begin{gathered}
\delta_{n}^{2}(\omega)=\int R_{n}^{2}(\omega) d \mu_{\omega}=\sum_{i \geq n} \frac{\int \psi_{\sigma^{i} \omega}^{2} \circ F_{\omega}^{i} d \mu_{\omega}}{\eta_{i}^{4 \gamma}(\omega)}=\sum_{i \geq n} \frac{\eta_{i}^{2}(\omega)-\eta_{i-1}^{2}(\omega)}{\eta_{i}^{4 \gamma}(\omega)} \\
\leq \int_{\eta_{n-1}^{2}(\omega)}^{\infty} \frac{1}{x^{2 \gamma}} d x=\eta_{n-1}^{2-4 \gamma}(\omega) \rightarrow 0, \\
\delta_{n}^{2}(\omega)=\sum_{i \geq n} \frac{\eta_{i}^{2}(\omega)-\eta_{i-1}^{2}(\omega)}{\eta_{i}^{4 \gamma}(\omega)} \geq C_{\omega}^{-2 \gamma} \cdot \sum_{i \geq n} \frac{\eta_{i}^{2}(\omega)-\eta_{i-1}^{2}(\omega)}{\eta_{i-1}^{4 \gamma}(\omega)} \\
\geq C_{\omega}^{-2 \gamma} \cdot \int_{\eta_{n-1}^{2}(\omega)}^{\infty} \frac{1}{x^{2 \gamma}} d x=C_{\omega}^{-2 \gamma} \cdot \eta_{n-1}^{2-4 \gamma}(\omega) \rightarrow 0,
\end{gathered}
$$

this proved (5.4). Now by (5.5), we have

$$
\left(B_{\tau_{i}^{\omega}}^{\omega}-B_{\tau_{i+1}^{\omega}}^{\omega}\right) \cdot \eta_{i}^{2 \gamma}(\omega)=\psi_{\sigma^{i} \omega} \circ F_{\omega}^{i},
$$

then

$$
\begin{gathered}
\left|\sum_{i \leq n} \psi_{\sigma^{i} \omega} \circ F_{\omega}^{i}-B_{\eta_{n}^{2}(\omega)}^{\omega}\right|=\left|\sum_{i \leq n} \psi_{\sigma^{i} \omega} \circ F_{\omega}^{i}-\sum_{i \leq n}\left(B_{\delta_{i}^{2}}^{\omega}(\omega)-B_{\delta_{i+1}^{2}(\omega)}^{\omega}\right) \cdot \eta_{i}^{2 \gamma}(\omega)\right| \\
=\left|\sum_{i \leq n}\left[\left(B_{\tau_{i}^{\omega}}^{\omega}-B_{\tau_{i+1}^{\omega}}^{\omega}\right)-\left(B_{\delta_{i}^{2}(\omega)}^{\omega}-B_{\delta_{i+1}^{2}(\omega)}^{\omega}\right)\right] \cdot \eta_{i}^{2 \gamma}(\omega)\right| \\
=\left|\sum_{i \leq n}\left(B_{\tau_{i}^{\omega}}^{\omega}-B_{\delta_{i}^{2}(\omega)}^{\omega}\right) \cdot\left(\eta_{i}^{2 \gamma}(\omega)-\eta_{i-1}^{2 \gamma}(\omega)\right)-\left(B_{\tau_{n+1}^{\omega}}^{\omega}-B_{\delta_{n+1}^{2}(\omega)}^{\omega}\right) \cdot \eta_{n}^{2 \gamma}(\omega)\right|
\end{gathered}
$$

By $\frac{1-\epsilon^{2}}{2}$-locally Hölder continuity of Brownian motion, the above identity becomes: there is $C_{\omega, \epsilon}>0$ s.t.

$$
\leq C_{\omega, \epsilon} \cdot\left[\sum_{i \leq n}\left|\tau_{i}^{\omega}-\delta_{i}^{2}(\omega)\right|^{\frac{1-\epsilon^{2}}{2}} \cdot\left(\eta_{i}^{2 \gamma}(\omega)-\eta_{i-1}^{2 \gamma}(\omega)\right)+\left|\tau_{n+1}^{\omega}-\delta_{n+1}^{2}(\omega)\right|^{\frac{1-\epsilon^{2}}{2}} \cdot \eta_{n}^{2 \gamma}(\omega)\right]
$$

By (5.8), the above inequality becomes:

$$
\leq C_{\omega, \epsilon} \cdot O\left(\left[\sum_{i \leq n}\left|\delta_{i}^{2(1+\epsilon)}(\omega)\right|^{\frac{1-\epsilon^{2}}{2}} \cdot\left(\eta_{i}^{2 \gamma}(\omega)-\eta_{i-1}^{2 \gamma}(\omega)\right)+\left|\delta_{n+1}^{2(1+\epsilon)}(\omega)\right|^{\frac{1-\epsilon^{2}}{2}} \cdot \eta_{n}^{2 \gamma}(\omega)\right]\right)
$$


Recall $\eta_{n}^{2}(\omega)=C_{\omega}^{ \pm} \cdot n, \delta_{n}^{2}(\omega) \leq \eta_{n-1}^{2-4 \gamma}(\omega)$ and $\eta_{n+1}^{2}(\omega)=C_{\omega}^{ \pm} \cdot \eta_{n}^{2}(\omega)$, the above inequality becomes:

$$
\begin{gathered}
\leq C_{\omega, \epsilon} \cdot O\left(\left[\sum_{i \leq n} \eta_{i-1}^{(2-4 \gamma) \cdot(1+\epsilon) \cdot \frac{1-\epsilon^{2}}{2}}(\omega) \cdot\left(\eta_{i}^{2 \gamma}(\omega)-\eta_{i-1}^{2 \gamma}(\omega)\right)+\eta_{n}^{(2-4 \gamma) \cdot(1+\epsilon) \cdot \frac{1-\epsilon^{2}}{2}}(\omega) \cdot \eta_{n}^{2 \gamma}(\omega)\right]\right) \\
\leq C_{\omega, \epsilon} \cdot O\left(\left[\sum_{i \leq n} \eta_{i}^{(2-4 \gamma) \cdot(1+\epsilon) \cdot \frac{1-\epsilon^{2}}{2}}(\omega) \cdot\left(\eta_{i}^{2 \gamma}(\omega)-\eta_{i-1}^{2 \gamma}(\omega)\right)+\eta_{n}^{(2-4 \gamma) \cdot(1+\epsilon) \cdot \frac{1-\epsilon^{2}}{2}}(\omega) \cdot \eta_{n}^{2 \gamma}(\omega)\right]\right) \\
\leq C_{\omega, \epsilon} \cdot O\left(\left[\int_{0}^{\eta_{n}^{2 \gamma}(\omega)} x^{\frac{(2-4 \gamma) \cdot(1+\epsilon) \cdot \frac{1-\epsilon^{2}}{2}}{2 \gamma}} d x+\eta_{n}^{2 \gamma+(2-4 \gamma) \cdot(1+\epsilon) \cdot \frac{1-\epsilon^{2}}{2}}(\omega)\right]\right) \\
\leq O\left(n^{\gamma-(2 \gamma-1) \cdot(1+\epsilon) \cdot\left(\frac{1-\epsilon^{2}}{2}\right)}\right)=O\left(n^{\frac{1}{4}+\frac{3 \epsilon-2 \epsilon^{3}-\epsilon^{2}}{4}}\right) .
\end{gathered}
$$

\section{Prove QASIP for Martingale}

Define $R_{n}(\omega):=\sum_{i \geq n} \frac{\psi_{\sigma^{i} \omega} \circ F_{\omega}^{i}}{\eta_{i}^{2 \gamma}(\omega)}$. By Lemma 5.3, we will verify (5.8):

$$
\tau_{n}^{\omega}-\delta_{n}^{2}(\omega)=O\left(\delta_{n}^{2(1+\epsilon)}(\omega)\right)
$$

\section{Lemma 5.4 (Stopping Time Decomposition)}

We have the decomposition:

$$
\tau_{n}^{\omega}-\delta_{n}^{2}(\omega):=R_{n}^{\prime}(\omega)+R_{n}^{\prime \prime}(\omega)+S_{n}^{\prime}(\omega)
$$

where

$$
\begin{gathered}
S_{n}^{\prime}(\omega)=\sum_{i \geq n}\left(\frac{\psi_{\sigma^{i} \omega}^{2} \circ F_{\omega}^{i}}{\eta_{i}^{4 \gamma}(\omega)}-\mathbb{E}_{\mu_{\omega}} \frac{\psi_{\sigma^{i} \omega}^{2} \circ F_{\omega}^{i}}{\eta_{i}^{4 \gamma}(\omega)}\right), \\
R_{n}^{\prime}(\omega):=\sum_{i \geq n} \tau_{i}^{\omega}-\tau_{i+1}^{\omega}-\mathbb{E}_{\mu_{\omega}}^{\left(F_{\omega}^{i+1}\right)^{-1} \mathcal{B}_{\sigma^{i+1} \omega}} \frac{\psi_{\sigma^{i} \omega}^{2} \circ F_{\omega}^{i}}{\eta_{i}^{4 \gamma}(\omega)}, \\
R_{n}^{\prime \prime}(\omega):=\sum_{i \geq n} \mathbb{E}_{\mu_{\omega}}^{\left(F_{\omega}^{i+1}\right)^{-1} \mathcal{B}_{\sigma^{i+1}}} \frac{\psi_{\sigma^{i} \omega}^{2} \circ F_{\omega}^{i}}{\eta_{i}^{4 \gamma}(\omega)}-\frac{\psi_{\sigma^{i} \omega}^{2} \circ F_{\omega}^{i}}{\eta_{i}^{4 \gamma}(\omega)}
\end{gathered}
$$

$R^{\prime}(\omega), R^{\prime \prime}(\omega)$ are reverse martingales w.r.t. $\left(\left(F_{\omega}^{i}\right)^{-1} \mathcal{B}_{\sigma^{i} \omega}\right)_{i \geq 1}$ and $\left(\mathcal{G}_{i}^{\omega}\right)_{i \geq 1}$ respectively satisfying: for a.e. $\omega \in \Omega$,

$$
R_{n}^{\prime}(\omega)=O\left(\delta_{n}^{2+2 \epsilon}(\omega)\right), R_{n}^{\prime \prime}(\omega)=O\left(\delta_{n}^{2+2 \epsilon}(\omega)\right),
$$

where the constants in $O(\cdot)$ depend on $\omega, \epsilon$ and $x \in \Delta_{\omega}$ and $\epsilon:=\frac{2 p(1+\delta)^{2}}{(p-1)(D-2-\delta)}$ for sufficiently small $\delta$. 
Proof It is straightforward to verify the decomposition. To prove $R_{n}^{\prime}(\omega), R_{n}^{\prime \prime}(\omega)$ are reverse martingales, we will verify the following two conditions:

Firstly,

$$
R_{n}^{\prime}(\omega) \text { and } R_{n}^{\prime \prime}(\omega) \text { are measurable w.r.t. }\left(F_{\omega}^{n}\right)^{-1} \mathcal{B}_{\sigma^{n} \omega} \text { and } \mathcal{G}_{n}^{\omega} \text { respectively. }
$$

Secondly,

$$
\begin{gathered}
\mathbb{E}_{\mu_{\omega}}^{\mathcal{G}_{n+1}^{\omega}} R_{n}^{\prime}(\omega)=\mathbb{E}_{\mu_{\omega}}^{\mathcal{G}_{n+1}^{\omega}}\left(\tau_{n}^{\omega}-\tau_{n+1}^{\omega}\right)-\mathbb{E}_{\mu_{\omega}}^{\mathcal{G}_{n+1}^{\omega}} \mathbb{E}_{\mu_{\omega}}^{\left(F_{\omega}^{n+1}\right)^{-1} \mathcal{B}_{\sigma^{n+1}}} \frac{\psi_{\sigma^{n} \omega}^{2} \circ F_{\omega}^{n}}{\eta_{n}^{4 \gamma}(\omega)} \\
+\mathbb{E}_{\mu_{\omega}}^{\mathcal{G}_{n+1}^{\omega}} \sum_{i \geq n+1} \tau_{i}^{\omega}-\tau_{i+1}^{\omega}-\mathbb{E}_{\mu_{\omega}}^{\left(F_{\omega}^{i+1}\right)^{-1} \mathcal{B}_{\sigma^{i+1}} \omega} \frac{\psi_{\sigma^{i} \omega}^{2} \circ F_{\omega}^{i}}{\eta_{i}^{4 \gamma}(\omega)} .
\end{gathered}
$$

By (5.6) and deceasing filtrations $\left(\left(F_{\omega}^{n}\right)^{-1} \mathcal{B}_{\sigma^{n} \omega}\right)_{n \geq 0}$ and $\left(\mathcal{G}_{n}^{\omega}\right)_{n \geq 0}$ satisfying $\left(F_{\omega}^{n}\right)^{-1} \mathcal{B}_{\sigma^{n} \omega} \subseteq$ $\mathcal{G}_{n}^{\omega}$, the equality above becomes:

$$
\begin{aligned}
& =\mathbb{E}_{\mu_{\omega}}^{\left(F_{\omega}^{n+1}\right)^{-1} \mathcal{B}_{\sigma^{n+1}}{ }} \frac{\psi_{\sigma^{n} \omega}^{2} \circ F_{\omega}^{n}}{\eta_{n}^{4 \gamma}(\omega)}-\mathbb{E}_{\mu_{\omega}}^{\left(F_{\omega}^{n+1}\right)^{-1} \mathcal{B}_{\sigma^{n+1}}} \frac{\psi_{\sigma^{n} \omega}^{2} \circ F_{\omega}^{n}}{\eta_{n}^{4 \gamma}(\omega)} \\
& +\mathbb{E}_{\mu_{\omega}}^{\mathcal{G}_{n+1}^{\omega}} \sum_{i \geq n+1} \tau_{i}^{\omega}-\tau_{i+1}^{\omega}-\mathbb{E}_{\mu_{\omega}}^{\left(F_{\omega}^{i+1}\right)^{-1} \mathcal{B}_{\sigma^{i+1}} \omega} \frac{\psi_{\sigma^{i} \omega}^{2} \circ F_{\omega}^{i}}{\eta_{i}^{4 \gamma}(\omega)}=R_{n+1}^{\prime \prime}(\omega) .
\end{aligned}
$$

Since $\left(\left(F_{\omega}^{n}\right)^{-1} \mathcal{B}_{\sigma^{n} \omega}\right)_{n \geq 0}$ is decreasing filtration,

$$
\begin{gathered}
\mathbb{E}_{\mu_{\omega}}^{\left(F_{\omega}^{n+1}\right)^{-1} \mathcal{B}_{\sigma^{n+1} \omega}} R_{n}^{\prime \prime}(\omega)=\mathbb{E}_{\mu_{\omega}}^{\left(F_{\omega}^{n+1}\right)^{-1} \mathcal{B}_{\sigma^{n+1}} \omega} \frac{\psi_{\sigma^{n}}^{2} \circ F_{\omega}^{n}}{\eta_{n}^{4 \gamma}(\omega)}-\mathbb{E}_{\mu_{\omega}}^{\left(F_{\omega}^{n+1}\right)^{-1} \mathcal{B}_{\sigma^{n+1}} \omega} \frac{\psi_{\sigma^{n} \omega}^{2} \circ F_{\omega}^{n}}{\eta_{n}^{4 \gamma}(\omega)} \\
+\mathbb{E}_{\mu_{\omega}}^{\left(F_{\omega}^{n+1}\right)^{-1} \mathcal{B}_{\sigma^{n+1}}} \sum_{i \geq n+1} \mathbb{E}_{\mu_{\omega}}^{\left(F_{\omega}^{i+1}\right)^{-1} \mathcal{B}_{\sigma^{i+1}}} \frac{\psi_{\sigma^{i} \omega}^{2} \circ F_{\omega}^{i}}{\eta_{i}^{4 \gamma}(\omega)}-\frac{\psi_{\sigma^{i} \omega}^{2} \circ F_{\omega}^{i}}{\eta_{i}^{4 \gamma}(\omega)}=R_{n+1}^{\prime \prime}(\omega) .
\end{gathered}
$$

Therefore $R^{\prime}(\omega), R^{\prime \prime}(\omega)$ are reverse martingales w.r.t. $\left(\left(F_{\omega}^{i}\right)^{-1} \mathcal{B}_{\sigma^{i} \omega}\right)_{i \geq 1}$ and $\left(\mathcal{G}_{i}^{\omega}\right)_{i \geq 1}$ respectively.

To prove the last statement, let $q:=\frac{(p-1)(D-2-\delta)}{2 p(1+\delta)}>2, \epsilon:=\frac{2 p(1+\delta)^{2}}{(p-1)(D-2-\delta)} \in\left(0, \frac{1}{2}\right)$ for sufficiently small $\delta$.

By Burkholder-Davis-Gundy inequality and Minkowski inequality, there is constant $C_{q}$ s.t.

$$
\left\|R_{n}^{\prime}(\omega)\right\|_{L^{q}\left(\mu_{\omega}\right)} \leq C_{q} \cdot \sqrt{\| \sum_{i \geq n} \mid \tau_{i}^{\omega}-\tau_{i+1}^{\omega}-\mathbb{E}_{\mu_{\omega}}^{\left.\left(F_{\omega}^{i+1}\right)^{-1} \mathcal{B}_{\sigma^{i+1}} \frac{\psi_{\sigma^{i} \omega}^{2} \circ F_{\omega}^{i}}{\eta_{i}^{4 \gamma}(\omega)}\right|^{2} \|_{L^{\frac{q}{2}}\left(\mu_{\omega}\right)}}}
$$




$$
\begin{aligned}
& \leq C_{q} \cdot \sqrt{\sum_{i \geq n}\left\|\tau_{i}^{\omega}-\tau_{i+1}^{\omega}-\left.\mathbb{E}_{\mu_{\omega}}^{\left(F_{\omega}^{i+1}\right)^{-1} \mathcal{B}_{\sigma^{i+1}} \omega} \frac{\psi_{\sigma^{i} \omega}^{2} \circ F_{\omega}^{i}}{\eta_{i}^{4 \gamma}(\omega)}\right|^{2}\right\|_{L^{\frac{q}{2}}\left(\mu_{\omega}\right)}} \\
& \leq \sqrt{2} \cdot C_{q} \cdot \sqrt{\sum_{i \geq n}\left\||| \tau_{i}^{\omega}-\left.\tau_{i+1}^{\omega}\right|^{2}+\mathbb{E}_{\mu_{\omega}}^{\left(F_{\omega}^{i+1}\right)^{-1} \mathcal{B}_{\sigma^{i+1}} \omega} \frac{\psi_{\sigma^{i} \omega}^{4} \circ F_{\omega}^{i}}{\eta_{i}^{8 \gamma}(\omega)}\right\|_{L^{\frac{q}{2}}\left(\mu_{\omega}\right)}}
\end{aligned}
$$

by (5.7), the above inequality becomes

$$
\leq 2 \cdot C_{q} \cdot \sqrt{\sum_{i \geq n}\left\|\frac{\psi_{\sigma^{i} \omega}^{4} \circ F_{\omega}^{i}}{\eta_{i}^{8 \gamma}(\omega)}\right\|_{L^{\frac{q}{2}}\left(\mu_{\omega}\right)}}=2 \cdot C_{q} \cdot \sqrt{\sum_{i \geq n} \frac{\left\|\psi_{\sigma^{i} \omega}^{4} \circ F_{\omega}^{i}\right\|_{L^{\frac{q}{2}}\left(\mu_{\omega}\right)}}{\eta_{i}^{8 \gamma}(\omega)}}
$$

Let $K_{n}(\omega):=\sum_{i \leq n}\left\|\psi_{\sigma^{i} \omega}^{4} \circ F_{\omega}^{i}\right\|_{L^{\frac{q}{2}\left(\mu_{\omega}\right)}}$, then by Lemma 5.1 and $\frac{2}{q}<1$,

$$
\mathbb{E}\left\|\psi_{\omega}^{4}\right\|_{L^{\frac{q}{2}\left(\mu_{\omega}\right)}} \leq\left(\mathbb{E} \int \psi_{\omega}^{2 q} d \mu_{\omega}\right)^{\frac{2}{q}}<\infty
$$

By ergodic theorem, for a.e. $\omega \in \Omega$, there is $C_{\omega} \geq 1$ such that $K_{n}(\omega)=C_{\omega}^{ \pm} \cdot n$ for all $n \in \mathbb{N}$. Then the above inequality becomes:

$$
\begin{gathered}
=2 \cdot C_{q} \cdot \sqrt{\sum_{i \geq n} \frac{K_{i}(\omega)-K_{i-1}(\omega)}{\eta_{i}^{8 \gamma}(\omega)}} \leq 2 \cdot C_{q} \cdot \sqrt{\frac{K_{n-1}(\omega)}{\eta_{n}^{8 \gamma}(\omega)}+\sum_{i \geq n} K_{i}(\omega) \cdot \frac{\left(\eta_{i+1}^{8 \gamma}(\omega)-\eta_{i}^{8 \gamma}(\omega)\right)}{\eta_{i}^{16 \gamma}(\omega)}} \\
\leq C_{\omega, q} \cdot \sqrt{\frac{n}{n^{4 \gamma}}+\int_{\eta_{n}^{8}(\omega)}^{\infty} \frac{1}{x^{\frac{16 \gamma-2}{8 \gamma}}} d x} \leq \sqrt{2} C_{\omega, q} \cdot \frac{1}{n^{\frac{4 \gamma-1}{2}}} .
\end{gathered}
$$

So by (5.4) and $\gamma=\frac{1}{4 \epsilon}$, there is $C_{\omega, q, \gamma}>0$ s.t.

$$
\left\|\frac{R_{n}^{\prime}(\omega)}{\delta_{n}^{2+2 \epsilon}(\omega)}\right\|_{L^{q}\left(\mu_{\omega}\right)} \leq C_{\omega, q, \gamma} \cdot \frac{\eta_{n}^{(4 \gamma-2)(1+\epsilon)}(\omega)}{n^{\frac{4 \gamma-1}{2}}} \leq C_{\omega, q, \gamma} \cdot \frac{n^{(2 \gamma-1)(1+\epsilon)}}{n^{\frac{4 \gamma-1}{2}}}=C_{\omega, q, \gamma} \cdot \frac{1}{n^{\epsilon}}
$$

So

$$
\left\|\frac{R_{n}^{\prime}(\omega)}{\delta_{n}^{2+2 \epsilon}(\omega)}\right\|_{L^{q}\left(\mu_{\omega}\right)}^{q} \leq C_{\omega, q, \gamma}^{q} \cdot \frac{1}{n^{q \epsilon}}=C_{\omega, q, \gamma}^{q} \frac{1}{n^{\frac{(p-1)(D-2-\delta)}{2 p(1+\delta)} \cdot \frac{2 p(1+\delta)^{2}}{(p-1)(D-2-\delta)}}}=C_{\omega, q, \gamma}^{q} \frac{1}{n^{1+\delta}}
$$

By Borel-Cantelli Lemma,

$$
R_{n}^{\prime}(\omega)=O\left(\delta_{n}^{2+2 \epsilon}(\omega)\right) \text { a.s.. }
$$

The estimate for $R_{n}^{\prime \prime}(\omega)$ is similar. 
Lemma 5.5 (Estimate $S_{n}^{\prime}(\omega)$ )

Define $S_{n}(\omega):=\sum_{i \leq n}\left(\psi_{\sigma^{i} \omega}^{2} \circ F_{\omega}^{i}-\int \psi_{\sigma^{i} \omega}^{2} \circ F_{\omega}^{i} d \mu_{\omega}\right)$. If

$$
S_{n}(\omega)=O\left(\eta_{n}^{2-(4 \gamma-2) \epsilon}(\omega)\right)=O\left(n^{\frac{1}{2}+\epsilon}\right) \text { a.s. }-\mu_{\omega},
$$

then

$$
S_{n}^{\prime}(\omega)=O\left(\delta_{n}^{2+2 \epsilon}(\omega)\right) \text { a.s. }-\mu_{\omega} .
$$

\section{Proof}

$$
\begin{aligned}
S_{n}^{\prime}(\omega) & =\sum_{i \geq n} \frac{S_{i}(\omega)-S_{i-1}(\omega)}{\eta_{i}^{4 \gamma}(\omega)}=-\frac{S_{n-1}(\omega)}{\eta_{n}^{4 \gamma}(\omega)}+\sum_{i \geq n} S_{i}(\omega) \cdot\left(\frac{1}{\eta_{i}^{4 \gamma}(\omega)}-\frac{1}{\eta_{i+1}^{4 \gamma}(\omega)}\right) \\
& \leq \frac{O\left(\eta_{n}^{2-(4 \gamma-2) \epsilon}(\omega)\right)}{\eta_{n}^{4 \gamma}(\omega)}+\sum_{i \geq n} O\left(\eta_{i}^{2-(4 \gamma-2) \epsilon}(\omega)\right) \cdot \frac{\eta_{i+1}^{4 \gamma}(\omega)-\eta_{i}^{4 \gamma}(\omega)}{\eta_{i}^{8 \gamma}(\omega)} \\
& \leq O\left(\frac{1}{\eta_{n}^{(4 \gamma-2)(1+\epsilon)}(\omega)}\right)+O\left(\int_{\eta_{n}^{4 \gamma}(\omega)}^{\infty} \frac{1}{x^{\frac{8 \gamma-2+(4 \gamma-2) \epsilon}{4 \gamma}}} d x\right)=O\left(\delta_{n}^{2(1+\epsilon)}(\omega)\right) .
\end{aligned}
$$

To estimate $S_{n}^{\prime}(\omega)$, we just need to estimate $S_{n}(\omega)$ : since

$$
\begin{gathered}
\sum_{i \leq n} \psi_{\sigma^{i-1} \omega}^{2} \circ F_{\omega}^{i-1}=\sum_{i \leq n}\left(\phi_{\sigma^{i} \omega} \circ F_{\omega}^{i}-g_{\sigma^{i} \omega} \circ F_{\omega}^{i}+g_{\sigma^{i-1} \omega} \circ F_{\omega}^{i-1}\right)^{2} \\
=\sum_{i \leq n} \phi_{\sigma^{i} \omega}^{2} \circ F_{\omega}^{i}+g_{\sigma^{i} \omega}^{2} \circ F_{\omega}^{i}+g_{\sigma^{i-1} \omega}^{2} \circ F_{\omega}^{i-1}+2 \phi_{\sigma^{i} \omega} \circ F^{i} \cdot g_{\sigma^{i-1} \omega} \circ F_{\omega}^{i-1} \\
\quad-2 \phi_{\sigma^{i} \omega} \circ F_{\omega}^{i} \cdot g_{\sigma^{i} \omega} \circ F_{\omega}^{i}-2 g_{\sigma^{i-1} \omega} \circ F_{\omega}^{i-1} \cdot g_{\sigma^{i} \omega} \circ F_{\omega}^{i} \\
=\sum_{i \leq n} \phi_{\sigma^{i} \omega}^{2} \circ F_{\omega}^{i}-g_{\sigma^{i} \omega}^{2} \circ F_{\omega}^{i}+g_{\sigma^{i-1} \omega}^{2} \circ F_{\omega}^{i-1}+2 \phi_{\sigma^{i} \omega} \circ F_{\omega}^{i} \cdot g_{\sigma^{i-1} \omega} \circ F_{\omega}^{i-1} \\
\quad+2 g_{\sigma^{i} \omega}^{2} \circ F_{\omega}^{i}-2 \phi_{\sigma^{i} \omega} \circ F_{\omega}^{i} \cdot g_{\sigma^{i} \omega} \circ F_{\omega}^{i}-2 g_{\sigma^{i-1} \omega} \circ F_{\omega}^{i-1} \cdot g_{\sigma^{i} \omega} \circ F_{\omega}^{i} \\
=\sum_{i \leq n} \phi_{\sigma^{i} \omega}^{2} \circ F_{\omega}^{i}-g_{\sigma^{i} \omega}^{2} \circ F_{\omega}^{i}+g_{\sigma^{i-1} \omega}^{2} \circ F_{\omega}^{i-1}+2 \phi_{\sigma^{i} \omega} \circ F_{\omega}^{i} \cdot g_{\sigma^{i-1} \omega} \circ F_{\omega}^{i-1} \\
\quad-2 \psi_{\sigma^{i-1} \omega} \circ F_{\omega}^{i-1} \cdot g_{\sigma^{i} \omega} \circ F_{\omega}^{i} \\
=\sum_{i \leq n} \phi_{\sigma^{i} \omega}^{2} \circ F_{\omega}^{i}-g_{\sigma^{n} \omega}^{2} \circ F_{\omega}^{n}+g_{\omega}^{2}+2 \sum_{i \leq n} \phi_{\sigma^{i} \omega} \circ F_{\omega}^{i} \cdot g_{\sigma^{i-1} \omega} \circ F_{\omega}^{i-1} \\
\quad-2 \sum_{i \leq n} \psi_{\sigma^{i-1} \omega} \circ F_{\omega}^{i-1} \cdot g_{\sigma^{i} \omega} \circ F_{\omega}^{i},
\end{gathered}
$$


So we have

$$
\begin{gathered}
S_{n-1}(\omega)=\sum_{i \leq n}\left(\phi_{\sigma^{i} \omega}^{2} \circ F_{\omega}^{i}-\mathbb{E}_{\mu_{\omega}} \phi_{\sigma^{i} \omega}^{2} \circ F_{\omega}^{i}\right) \\
+2 \sum_{i \leq n}\left(\phi_{\sigma^{i} \omega} \circ F_{\omega}^{i} \cdot g_{\sigma^{i-1} \omega} \circ F_{\omega}^{i-1}-\int \phi_{\sigma^{i} \omega} \circ F_{\omega}^{i} \cdot g_{\sigma^{i-1} \omega} \circ F_{\omega}^{i-1} d \mu_{\omega}\right) \\
-2 \sum_{i \leq n}\left(\psi_{\sigma^{i-1} \omega} \circ F_{\omega}^{i-1} \cdot g_{\sigma^{i} \omega} \circ F_{\omega}^{i}-\int \psi_{\sigma^{i-1} \omega} \circ F_{\omega}^{i-1} \cdot g_{\sigma^{i} \omega} \circ F_{\omega}^{i} d \mu_{\omega}\right) \\
-g_{\sigma^{n} \omega}^{2} \circ F_{\omega}^{n}+g_{\omega}^{2}+\mathbb{E}_{\mu_{\omega}} g_{\sigma^{n} \omega}^{2} \circ F_{\omega}^{n}-\mathbb{E}_{\mu_{\omega}} g_{\omega}^{2} \\
\int \psi_{\sigma^{i-1} \omega} \circ F_{\omega}^{i-1} \cdot g_{\sigma^{i} \omega} \circ F_{\omega}^{i} d \mu_{\omega}=\int g_{\sigma^{i} \omega} \circ F_{\omega}^{i} \cdot \mathbb{E}_{\mu_{\omega}}\left[\psi_{\sigma^{i-1} \omega} \circ F_{\omega}^{i-1} \mid\left(F_{\omega}^{i}\right)^{-1} \mathcal{B}_{\sigma^{i} \omega}\right] d \mu_{\omega}=0
\end{gathered}
$$

the above equality becomes

$$
\begin{gathered}
=\sum_{i \leq n}\left(\phi_{\sigma^{i} \omega}^{2} \circ F_{\omega}^{i}-\mathbb{E}_{\mu_{\omega}} \phi_{\sigma^{i} \omega}^{2} \circ F_{\omega}^{i}\right) \\
+2 \sum_{i \leq n}\left(\phi_{\sigma^{i} \omega} \circ F_{\omega}^{i} \cdot g_{\sigma^{i-1} \omega} \circ F_{\omega}^{i-1}-\int \phi_{\sigma^{i} \omega} \circ F_{\omega}^{i} \cdot g_{\sigma^{i-1} \omega} \circ F_{\omega}^{i-1} d \mu_{\omega}\right) \\
-2 \sum_{i \leq n}\left(\psi_{\sigma^{i-1} \omega} \circ F_{\omega}^{i-1} \cdot g_{\sigma^{i} \omega} \circ F_{\omega}^{i}\right) \\
-g_{\sigma^{n} \omega}^{2} \circ F_{\omega}^{n}+g_{\omega}^{2}+\mathbb{E}_{\mu_{\omega}} g_{\sigma^{n} \omega}^{2} \circ F_{\omega}^{n}-\mathbb{E}_{\mu_{\omega}} g_{\omega}^{2}
\end{gathered}
$$

To estimate $S_{n}(\omega)$, we will estimate $(5.9),(5.10),(5.11),(5.12)$.

\section{Lemma 5.6 (Estimate (5.12))}

Let $\epsilon=\max \left\{\frac{2(2+\delta)(1+\delta) p}{(D-2)(p-1)}-\frac{1}{2}, 0\right\} \in\left(0, \frac{1}{2}\right)$, for sufficiently small $\delta>0$, a.e. $\omega \in \Omega$,

$$
(5.12)=O_{\omega, x, \delta}\left(n^{\frac{1}{2}+\epsilon}\right) \text { a.s. }-\mu_{\omega} .
$$

Proof For sufficiently small $\delta, \frac{2(2+\delta)(1+\delta) p}{(D-2-\delta)(p-1)} \leq \epsilon+\frac{1}{2}$. By Lemma 5.1,

$(5.12)=O_{\omega, x, \delta}\left(n^{\frac{2(2+\delta)(1+\delta) p}{(D-2-\delta)(p-1)}}\right)+O_{\omega, x}(1)+O_{\omega, x, \delta}\left(n^{\frac{2(1+\delta) p}{(D-2-\delta)(p-1)}}\right)+O_{\omega}(1)=O_{\omega, x, \delta}\left(n^{\frac{1}{2}+\epsilon}\right)$.

\section{Lemma 5.7 (Estimate (5.9))}

Let $\epsilon=\frac{p(1+\delta)^{2}}{(p-1)(D-2-\delta)}$, for sufficiently small $\delta>0$, a.e. $\omega \in \Omega$,

$$
(5.9)=O_{\omega, x, \delta}\left(n^{\frac{1}{2}+\epsilon}\right) .
$$


Proof Let $q=\frac{(D-2-\delta)(p-1)}{(1+\delta) p}$, since $\phi_{(\cdot)}^{2}-\mathbb{E}_{\mu_{(\cdot)}} \phi_{(\cdot)}^{2} \in L^{\infty}(\Delta, \mu) \bigcap \mathcal{F}_{\beta, p}^{\mathcal{K}} \subseteq L^{q}(\Delta, \mu)$ with Lipschitz constant $2 C_{\phi}^{2}$, then by Lemma 3.5 and (3.6), there is $C_{\phi, q, p, h, F}>0$,

$$
\begin{gathered}
\left(\mathbb{E} \int\left|P_{\omega}^{n}\left(\phi_{\omega}^{2}-\mathbb{E}_{\mu_{\omega}} \phi_{\omega}^{2}\right)\right|^{q} d \mu_{\omega}\right)^{\frac{1}{q}} \leq\left(2 C_{\phi}^{2}\right)^{\frac{q-1}{q}} \cdot\left(\mathbb{E} \int\left|P_{\omega}^{n}\left(\phi_{\omega}^{2}-\mathbb{E}_{\mu_{\omega}} \phi_{\omega}^{2}\right)\right| d \mu_{\omega}\right)^{\frac{1}{q}} \\
\leq C_{\phi, q, p, h, F} \cdot \frac{1}{n^{\frac{(D-2-\delta)(p-1)}{q p}}} \leq C_{\phi, q, p, h, F} \cdot \frac{1}{n^{1+\delta}}<\infty .
\end{gathered}
$$

So by Lemma 4.3 , for a.e. $\omega \in \Omega$,

$$
(5.9)=O_{\omega, x, \delta}\left(n^{\frac{1}{2}+\frac{1+\delta}{q}}\right)=O_{\omega, x, \delta}\left(n^{\frac{1}{2}+\epsilon}\right) .
$$

\section{Lemma 5.8 (Estimate (5.11))}

For sufficiently small $\delta$, let $\epsilon=\frac{2 p(1+\delta)^{2}}{(p-1)(D-2-\delta)} \in\left(0, \frac{1}{2}\right)$, for a.e. $\omega \in \Omega$,

$$
\sum_{i \leq n}\left(\psi_{\sigma^{i-1} \omega} \circ F_{\omega}^{i-1} \cdot g_{\sigma^{i} \omega} \circ F_{\omega}^{i}\right)=O_{\omega, x, \delta}\left(n^{\frac{1}{2}+\epsilon}\right) \text { a.s. }-\mu_{\omega} .
$$

Proof From Lemma 5.1, for a.e. $\omega \in \Omega,\left(\psi_{\sigma^{i} \omega} \circ F_{\omega}^{i}\right)_{i \geq 0}$ is reverse martingale difference, so is $\left(\psi_{\sigma^{i-1} \omega} \circ F_{\omega}^{i-1} \cdot g_{\sigma^{i} \omega} \circ F_{\omega}^{i}\right)_{i \geq 1}$. Let $q=\frac{(D-2-\delta)(p-1)}{2(1+\delta) p}$, then by Lemma 5.1 again and Hölder inequality,

$\left(\mathbb{E} \int\left|\psi_{\omega} \circ F_{\omega} \cdot g_{\sigma \omega} \circ F_{\omega}\right|^{q} d \mu_{\omega}\right)^{\frac{1}{q}} \leq\left(\mathbb{E} \int\left|g_{\sigma \omega} \circ F_{\omega}\right|^{2 q} d \mu_{\omega}\right)^{\frac{1}{2 q}} \cdot\left(\mathbb{E} \int\left|\psi_{\omega} \circ F_{\omega}\right|^{2 q} d \mu_{\omega}\right)^{\frac{1}{2 q}}<\infty$.

Then by Lemma 4.2,

$$
(5.11)=O_{x, \omega, q, \delta}\left(n^{\frac{1}{2}+\frac{1+\delta}{q}}\right)=O_{x, \omega, q, \delta}\left(n^{\frac{1}{2}+\epsilon}\right) \text { a.S. }-\mu_{\omega} .
$$

\section{Lemma 5.9 (Estimate (5.10))}

For sufficiently small $\delta$, let $\epsilon=\frac{2 p(1+\delta)^{2}}{(p-1)(D-2-\delta)} \in\left(0, \frac{1}{2}\right)$, for a.e. $\omega \in \Omega$,

$$
\sum_{i \leq n}\left(\phi_{\sigma^{i} \omega} \circ F_{\omega}^{i} \cdot g_{\sigma^{i-1} \omega} \circ F_{\omega}^{i-1}-\int \phi_{\sigma^{i} \omega} \circ F_{\omega}^{i} \cdot g_{\sigma^{i-1} \omega} \circ F_{\omega}^{i-1} d \mu_{\omega}\right)=O_{x, \omega, q, \delta}\left(n^{\frac{1}{2}+\epsilon}\right) \text { a.s. }-\mu_{\omega} .
$$


Proof For sufficiently small $\delta$, let $q=\frac{(D-2-\delta)(p-1)}{2(1+\delta) p}>2$, denote $\Phi_{\omega}:=\phi_{\sigma \omega} \circ F_{\omega}$. $g_{\omega}-\int \phi_{\sigma \omega} \circ F_{\omega} \cdot g_{\omega} d \mu_{\omega}$. Then

$$
\|\Phi\|_{L^{q}(\Delta, \mu)} \leq 2 C_{\phi} \cdot\|g\|_{L^{q}(\Delta, \mu)}<\infty
$$

Therefore, by (3.9) and Minkowski inequality,

$$
\begin{aligned}
& \left.\left(\mathbb{E} \int\left|P_{\omega}^{k}\left(\Phi_{\omega}\right)\right|^{q} d \mu_{\sigma^{k} \omega}\right)^{\frac{1}{q}}=\left(\mathbb{E} \int\left|P_{\omega}^{k}\left[\phi_{\sigma \omega} \circ F_{\omega} \cdot g_{\omega}-\int \phi_{\sigma \omega} \circ F_{\omega} \cdot g_{\omega} d \mu_{\omega}\right]\right|^{q} d \mu_{\sigma^{k} \omega}\right)\right)^{\frac{1}{q}} \\
& \left.\leq \sum_{i \geq 0}\left(\mathbb{E} \int\left|P_{\omega}^{k}\left[\phi_{\sigma \omega} \circ F_{\omega} \cdot P_{\sigma^{-i} \omega}^{i}\left(\phi_{\sigma^{-i} \omega}\right)-\int \phi_{\sigma \omega} \circ F_{\omega} \cdot P_{\sigma^{-i} \omega}^{i}\left(\phi_{\sigma^{-i} \omega}\right) d \mu_{\omega}\right]\right|^{q} d \mu_{\sigma^{k} \omega}\right)\right)^{\frac{1}{q}} \\
& \left.\leq \sum_{i \geq 0}\left(\mathbb{E} \int\left|P_{\sigma \omega}^{k-1}\left[\phi_{\sigma \omega} \cdot P_{\sigma^{-i} \omega}^{i+1}\left(\phi_{\sigma^{-i} \omega}\right)-\int \phi_{\sigma \omega} \cdot P_{\sigma^{-i} \omega}^{i+1}\left(\phi_{\sigma^{-i} \omega}\right) d \mu_{\sigma \omega}\right]\right|^{q} d \mu_{\sigma^{k} \omega}\right)\right)^{\frac{1}{q}} \\
& \left.\quad \leq \sum_{i<k}\left(\mathbb{E} \int\left|P_{\sigma \omega}^{k-1}\left[\phi_{\sigma \omega} \cdot P_{\sigma^{-i} \omega}^{i+1}\left(\phi_{\sigma^{-i} \omega}\right)-\int \phi_{\sigma \omega} \cdot P_{\sigma^{-i} \omega}^{i+1}\left(\phi_{\sigma^{-i} \omega}\right) d \mu_{\sigma \omega}\right]\right|^{q} d \mu_{\sigma^{k} \omega}\right)\right)^{\frac{1}{q}} \\
& \left.\quad+\sum_{i \geq k}\left(\mathbb{E} \int\left|P_{\sigma \omega}^{k-1}\left[\phi_{\sigma \omega} \cdot P_{\sigma^{-i} \omega}^{i+1}\left(\phi_{\sigma^{-i} \omega}\right)-\int \phi_{\sigma \omega} \cdot P_{\sigma^{-i} \omega}^{i+1}\left(\phi_{\sigma^{-i} \omega}\right) d \mu_{\sigma \omega}\right]\right|^{q} d \mu_{\sigma^{k} \omega}\right)\right)^{\frac{1}{q}} .
\end{aligned}
$$

By (3.6),

$$
\left\|\phi_{\sigma \omega} \cdot P_{\sigma^{-i} \omega}^{i+1}\left(\phi_{\sigma^{-i} \omega}\right)\right\|_{L^{\infty}\left(\mu_{\sigma \omega}\right)} \leq C_{\phi}^{2}
$$

then the above inequality becomes: there is $C_{\phi, q}>0$ s.t.

$$
\begin{aligned}
& \left.\leq C_{\phi, q} \cdot \sum_{i<k}\left(\mathbb{E} \int\left|P_{\sigma \omega}^{k-1}\left[\phi_{\sigma \omega} \cdot P_{\sigma^{-i} \omega}^{i+1}\left(\phi_{\sigma^{-i} \omega}\right)-\int \phi_{\sigma \omega} \cdot P_{\sigma^{-i} \omega}^{i+1}\left(\phi_{\sigma^{-i} \omega}\right) d \mu_{\sigma \omega}\right]\right| d \mu_{\sigma^{k} \omega}\right)\right)^{\frac{1}{q}} \\
& \left.+C_{\phi, q} \cdot \sum_{i \geq k}\left(\mathbb{E} \int\left|P_{\sigma \omega}^{k-1}\left[\phi_{\sigma \omega} \cdot P_{\sigma^{-i} \omega}^{i+1}\left(\phi_{\sigma^{-i} \omega}\right)-\int \phi_{\sigma \omega} \cdot P_{\sigma^{-i} \omega}^{i+1}\left(\phi_{\sigma^{-i} \omega}\right) d \mu_{\sigma \omega}\right]\right| d \mu_{\sigma^{k} \omega}\right)\right)^{\frac{1}{q}} \\
& \left.\leq C_{\phi, q} \cdot \sum_{i<k}\left(\mathbb{E} \int\left|P_{\sigma \omega}^{k-1}\left[\phi_{\sigma \omega} \cdot P_{\sigma^{-i} \omega}^{i+1}\left(\phi_{\sigma^{-i} \omega}\right)-\int \phi_{\sigma \omega} \cdot P_{\sigma^{-i} \omega}^{i+1}\left(\phi_{\sigma^{-i} \omega}\right) d \mu_{\sigma \omega}\right]\right| d \mu_{\sigma^{k} \omega}\right)\right)^{\frac{1}{q}}
\end{aligned}
$$




$$
\left.+C_{\phi, q} \cdot \sum_{i \geq k}\left(\mathbb{E} \int\left|P_{\sigma^{-i} \omega}^{i+1}\left(\phi_{\sigma^{-i} \omega}\right)\right| d \mu_{\sigma \omega}+\mathbb{E} \int\left|P_{\sigma^{-i} \omega}^{i+1}\left(\phi_{\sigma^{-i} \omega}\right)\right| d \mu_{\sigma \omega}\right] \mid\right)^{\frac{1}{q}}
$$

To proceed the estimate, we need to find the regularity of $\phi_{\sigma \omega} \cdot P_{\sigma^{-i} \omega}^{i+1}\left(\phi_{\sigma^{-i} \omega}\right)$ : for any $x, y \in \Delta_{\sigma \omega}$, by Lemma 4.4 and (3.6),

$$
\begin{gathered}
\left|\phi_{\sigma \omega}(x) \cdot P_{\sigma^{-i} \omega}^{i+1}\left(\phi_{\sigma^{-i} \omega}\right)(x)-\phi_{\sigma \omega}(y) \cdot P_{\sigma^{-i} \omega}^{i+1}\left(\phi_{\sigma^{-i} \omega}\right)(y)\right| \\
\leq\left|\phi_{\sigma \omega}(x)-\phi_{\sigma \omega}(y)\right| \cdot C_{\phi}+\left|P_{\sigma^{-i} \omega}^{i+1}\left(\phi_{\sigma^{-i} \omega}\right)(y)-P_{\sigma^{-i} \omega}^{i+1}\left(\phi_{\sigma^{-i} \omega}\right)(x)\right| \cdot C_{\phi} \\
\leq \mathcal{K}_{\sigma \omega} \cdot \beta^{s_{\sigma \omega}(x, y)} \cdot C_{\phi}^{2}+4 \mathcal{K}_{\sigma^{-i} \omega} \cdot \beta^{s_{\sigma \omega}(x, y)} \cdot C_{\phi}^{2} .
\end{gathered}
$$

Then $\phi_{(\cdot)} \cdot P_{\sigma^{-(i+1)(\cdot)}}^{i+1}\left(\phi_{\sigma^{-(i+1)}(\cdot)}\right) \in \mathcal{F}_{\beta, p}^{\left(\mathcal{K}+4 \mathcal{K} \circ \sigma^{-(i+1)}\right)}$ with Lipschitz constant $C_{\phi}^{2}$. So by Lemma 3.5, we can continuous our estimate: there is constant $C=C_{\phi}^{2} \cdot C_{h, F, \beta, \delta, p}$. $\left\|\mathcal{K}+4 \mathcal{K} \circ \sigma^{-(i+1)}\right\|_{L^{p}} \leq 5 C_{\phi}^{2} \cdot C_{h, F, \beta, \delta, p} \cdot\|\mathcal{K}\|_{L^{p}}$ s.t.

$$
\begin{gathered}
\leq C \cdot C_{\phi, q}^{\frac{1}{q}} \cdot \sum_{i<k} \frac{1}{(k-1)^{\frac{(D-2-\delta)(p-1)}{q p}}}+C \cdot C_{\phi, q}^{\frac{1}{q}} \cdot \sum_{i \geq k} \frac{1}{i^{\frac{(D-2-\delta)(p-1)}{q p}}} \\
\leq 2 C \cdot C_{\phi, q}^{\frac{1}{q}} \cdot \frac{1}{k^{\frac{(D-2-\delta)(p-1)}{q p}-1}}=2 C \cdot C_{\phi, q}^{\frac{1}{q}} \cdot \frac{1}{k^{2 \delta+1}} .
\end{gathered}
$$

By Lemma 4.3,

$$
\begin{gathered}
\sum_{i \leq n}\left(\phi_{\sigma^{i} \omega} \circ F_{\omega}^{i} \cdot g_{\sigma^{i-1} \omega} \circ F_{\omega}^{i-1}-\int \phi_{\sigma^{i} \omega} \circ F_{\omega}^{i} \cdot g_{\sigma^{i-1} \omega} \circ F_{\omega}^{i-1} d \mu_{\omega}\right) \\
=O_{x, \omega, q, \delta}\left(n^{\frac{1}{2}+\frac{1+\delta}{q}}\right)=O_{x, \omega, q, \delta}\left(n^{\frac{1}{2}+\epsilon}\right) \text { a.s. }-\mu_{\omega} .
\end{gathered}
$$

So we have the following summary:

Lemma 5.10 (QASIP for $\left.\left(\psi_{\sigma^{i} \omega} \circ F_{\omega}^{i}\right)_{i \geq 0}\right)$

For sufficiently small $\delta$, let $\epsilon=\frac{2 p(1+\delta)^{2}}{(p-1)(D-2-\delta)} \in\left(0, \frac{1}{2}\right)$, then for a.e. $\omega \in \Omega$,

$$
\left|\sum_{i \leq n} \psi_{\sigma^{i} \omega} \circ F_{\omega}^{i}-B_{\eta_{n}^{2}(\omega)}^{\omega}\right|=O\left(n^{\frac{1}{4}+\frac{3 \epsilon-2 \epsilon^{3}-\epsilon^{2}}{4}}\right) \text { a.s.. }
$$

Proof By Lemma 5.4, 5.5, 5.6, 5.7, 5.8, 5.9, for sufficiently small $\delta$, we have: for a.e. $\omega \in \Omega$,

$$
\tau_{n}^{\omega}-\delta_{n}^{2}(\omega)=O\left(\delta_{n}^{2+2 \epsilon}(\omega)\right) \text { a.s. },
$$

with $\epsilon=\max \left\{\frac{2 p(1+\delta)^{2}}{(p-1)(D-2-\delta)}, \max \left\{\frac{2(2+\delta)(1+\delta) p}{(D-2)(p-1)}-\frac{1}{2}, 0\right\}, \frac{p(1+\delta)^{2}}{(p-1)(D-2-\delta)}\right\}=\frac{2 p(1+\delta)^{2}}{(p-1)(D-2-\delta)}$. 
By Lemma 5.3,

$$
\left|\sum_{i \leq n} \psi_{\sigma^{i} \omega} \circ F_{\omega}^{i}-B_{\eta_{n}^{2}(\omega)}^{\omega}\right|=O\left(n^{\frac{1}{4}+\frac{3 \epsilon-2 \epsilon^{3}-\epsilon^{2}}{4}}\right) \text { a.s.. }
$$

Now we can prove the final statement (2.2) of QASIP:

\section{Lemma 5.11 (QASIP for $\left.\left(\phi_{\sigma^{i} \omega} \circ F_{\omega}^{i}\right)_{i \geq 0}\right)$}

then for a.e. $\omega \in \Omega$,

$$
\left|\sum_{i \leq n} \phi_{\sigma^{i} \omega} \circ F_{\omega}^{i}-B_{\sigma_{n}^{2}(\omega)}^{\omega}\right|=O\left(n^{\frac{1}{4}+\epsilon_{0}}\right) \text { a.s.. }
$$

1. if $\rho_{n}=e^{-a \cdot n^{b}}, \epsilon_{0}>0$ can be chosen to be any small number,

2. if $\rho_{n}=\frac{1}{n^{D}}, \epsilon_{0}$ can be chosen to be any number between $\left(\epsilon_{D}, \frac{1}{4}\right)$, where

$$
\epsilon_{D}=\max \left\{\frac{1}{4}+\frac{3 \epsilon_{1}-2 \epsilon_{1}^{3}-\epsilon_{1}^{2}}{4}, \epsilon_{1}, \frac{1+\epsilon_{1}}{4}\right\}-\frac{1}{4}
$$

and

$$
\epsilon_{1}=\frac{2 p}{(p-1)(D-2)} \in\left(0, \frac{1}{2}\right)
$$

Proof Since $\epsilon_{1}=\frac{2 p}{(p-1)(D-2)} \in\left(0, \frac{1}{2}\right)$, so there is sufficiently small $\delta$ s.t.

$$
\epsilon=\frac{2 p(1+\delta)^{2}}{(p-1)(D-2-\delta)} \in\left(\epsilon_{1}, \frac{1}{2}\right) .
$$

By Lemma 5.1 and Lemma 5.10,

$$
\begin{gathered}
\sum_{1 \leq i \leq n} \phi_{\sigma^{i} \omega} \circ F_{\omega}^{i}=\sum_{1 \leq i \leq n} \psi_{\sigma^{i-1} \omega} \circ F_{\omega}^{i-1}+g_{\sigma^{n} \omega} \circ F_{\omega}^{n}-g_{\omega}=\sum_{1 \leq i \leq n} \psi_{\sigma^{i-1} \omega} \circ F_{\omega}^{i-1} \\
+O_{\omega, x, \delta}\left(n^{\frac{(2+\delta)(1+\delta) p}{(D-2) \delta(p-1)}}\right)+O_{\omega, x}(1)=B_{\eta_{n-1}^{2}(\omega)}^{\omega}+O\left(n^{\frac{1}{4}+\frac{3 \epsilon-2 \epsilon^{3}-\epsilon^{2}}{4}}\right)+O_{\omega, x, \delta}\left(n^{\epsilon}\right)+O_{\omega, x}(1) \\
=B_{\eta_{n-1}^{2}(\omega)}^{\omega}+O\left(n^{\max \left\{\frac{1}{4}+\frac{3 \epsilon-2 \epsilon^{3}-\epsilon^{2}}{4}, \epsilon\right\}}\right) .
\end{gathered}
$$

By (5.2) and basic property of Brownian motion,

$$
B_{\eta_{n-1}^{2}(\omega)}^{\omega}=B_{\sigma_{n}^{2}(\omega)}^{\omega}+O\left(n^{\frac{1}{4}+\frac{(1+\delta)^{2} \cdot p}{2(D-2-\delta) \cdot(p-1)}}\right)=B_{\sigma_{n}^{2}(\omega)}^{\omega}+O\left(n^{\frac{1+\epsilon}{4}}\right) .
$$

Therefore, 


$$
\begin{aligned}
& \sum_{1 \leq i \leq n} \phi_{\sigma^{i} \omega} \circ F_{\omega}^{i}=B_{\sigma_{n}^{2}(\omega)}^{\omega}+O\left(n^{\frac{1+\epsilon}{4}}\right)+O\left(n^{\max \left\{\frac{1}{4}+\frac{3 \epsilon-2 \epsilon^{3}-\epsilon^{2}}{4}, \epsilon\right\}}\right) \\
& =B_{\sigma_{n}^{2}(\omega)}^{\omega}+O\left(n^{\max \left\{\frac{1}{4}+\frac{3 \epsilon-2 \epsilon^{3}-\epsilon^{2}}{4}, \epsilon, \frac{1+\epsilon}{4}\right\}}\right)=B_{\sigma_{n}^{2}(\omega)}^{\omega}+O\left(n^{\frac{1}{4}+\epsilon_{0}}\right),
\end{aligned}
$$

where $\epsilon_{0}=\max \left\{\frac{1}{4}+\frac{3 \epsilon-2 \epsilon^{3}-\epsilon^{2}}{4}, \epsilon, \frac{1+\epsilon}{4}\right\}-\frac{1}{4} \in\left(\epsilon_{D}, \frac{1}{4}\right)$.

If $\rho_{n}=e^{-a \cdot n^{b}}$, then $\rho_{n} \leq \frac{1}{n^{D}}$ for sufficiently large $D$. Then $\epsilon_{D}$ is arbitrary closed to 0 , so is $\epsilon_{0}$.

\section{Project From Tower}

In this section, we consider the RDS which can be extended to RYT:

\section{Definition 6.1 (Induced Random Markov Map)}

(1) Assume Bernoulli scheme $(\Omega, \mathbb{P}, \sigma):=\left(I^{\mathbb{Z}}, \nu^{\mathbb{Z}}, \sigma\right)$ where $I$ is compact interval with normalized Lebegues probability measure $\nu$. $(M$, Leb, $d)$ is compact Riemannian manifold with Riemannian volume Leb and Riemanian distant $d .\left(f_{\omega}\right)_{\omega \in \Omega}$ is nonsingular random transformations w.r.t. Leb on M. Define:

$$
f_{\omega}^{n}:=f_{\sigma^{n-1} \omega} \circ f_{\sigma^{n-2} \omega} \circ \cdots \circ f_{\sigma \omega} \circ f_{\omega}
$$

(2) Assume an open $\Lambda \subset M$, with normalized probability $m$ inherited from Leb.

(3) Assume for a.e. $\omega \in \Omega$, there are countable partition $\mathcal{P}_{\omega}$ of a full measure subset $\mathcal{D}_{\omega}$ of $\Lambda$ and function $R_{\omega}: \Lambda \rightarrow \mathbb{N}$ such that $R_{\omega}$ is constant on each $U_{\omega} \in \mathcal{P}_{\omega},\left\{x \in \Lambda: R_{\omega}(x)=n\right\}$ only depends on $\omega_{0}, \omega_{1}, \cdots, \omega_{n-1}$ and $\left.f_{\omega}^{R_{\omega}}\right|_{U_{\omega}}$ is diffeomorphism from $U_{\omega}$ to $\Lambda$.

(4) Assume there are $N \in \mathbb{N},\left\{\epsilon_{i}>0, i=1, \cdots, N\right\}$ and $\left\{t_{i} \in \mathbb{N}, i=1, \cdots, N\right\}$ with $\operatorname{gcd}\left(t_{i}\right)=1$ such that for a.e. $\omega \in \Omega$, all $1 \leq i \leq N$,

$$
\operatorname{Leb}\left(x \in \Lambda: R_{\omega}(x)=t_{i}\right)>\epsilon_{i}
$$

(5) Assume there are $\beta \in(0,1)$, constant $C>0$, random function $1 \leq \mathcal{K}_{(\cdot)} \in L^{p}(\Omega)$ s.t. for a.e. $\omega \in \Omega$, any $U_{\omega} \in \mathcal{P}_{\omega}, x, y \in U_{\omega}$, and $0 \leq k \leq\left. R_{\omega}\right|_{U_{\omega}}$ :

$$
\begin{gathered}
d\left(f_{\omega}^{R_{\omega}}(x), f_{\omega}^{R_{\omega}}(y)\right) \geq \beta^{-1} \cdot d(x, y), \\
\left|\log \frac{J f_{\omega}^{R_{\omega}}(x)}{J f_{\omega}^{R_{\omega}}(y)}\right| \leq C \cdot d\left(f_{\omega}^{R_{\omega}}(x), f_{\omega}^{R_{\omega}}(y)\right), \\
d\left(f_{\omega}^{k}(x), f_{\omega}^{k}(y)\right) \leq C \cdot \mathcal{K}_{\sigma^{k} \omega} \cdot d\left(f_{\omega}^{R_{\omega}}(x), f_{\omega}^{R_{\omega}}(y)\right) .
\end{gathered}
$$


(6) Assume there is constant $C>0$ s.t.

$$
\int \operatorname{Leb}\left(x \in \Lambda: R_{\omega}(x)>n\right) d \mathbb{P} \leq C \cdot \rho_{n}
$$

where $\rho_{n}:=e^{-a \cdot n^{b}}$ or $\frac{1}{n^{D}}$ for some constant $a>0, b \in(0,1], D>2+\frac{4 p}{p-\gamma}$, $\gamma \in(0,1]$ will be explained in Theorem 6.2 below.

\section{Theorem 6.2 (QASIP for RDS)}

Assume $\left(M,\left(f_{\omega}\right)_{\omega \in \Omega}\right.$, Leb) satisfies the conditions in Definition 6.1. Then for a.e. $\omega \in \Omega$, there are equivariant probability measures $\left(v_{\omega}\right)_{\omega \in \Omega}$ on $M$, that is,

$$
\left(f_{\omega}\right)_{*} v_{\omega}=v_{\sigma \omega}
$$

For any Hölder function $\phi$ on $M$ with Hölder exponent $\gamma \in(0,1]$. Define

$$
\begin{gathered}
\varphi_{\omega}:=\varphi-\int \varphi d v_{\omega}, \\
\sigma_{n}^{2}(\omega):=\int\left(\sum_{k \leq n} \varphi_{\sigma^{k} \omega} \circ f_{\omega}^{k}\right)^{2} d v_{\omega} .
\end{gathered}
$$

Then $\left(M,\left(f_{\omega}\right)_{\omega \in \Omega}\right)$ satisfies the following:

1. There is $\sigma^{2} \geq 0$ s.t. $\lim _{n \rightarrow \infty} \frac{\sigma_{n}^{2}(\omega)}{n}=\sigma^{2}$ a.e. $\omega \in \Omega$.

2. If $\sigma^{2}>0$, we have QASIP: there is $\epsilon_{0} \in\left(0, \frac{1}{4}\right)$ s.t. for a.e. $\omega \in \Omega$, there is Brownian motion $B^{\omega}$ defined on some extension of probability space $\left(M, v_{\omega}\right)$, say $\mathbf{M}_{\omega}$, such that:

$$
\sum_{k \leq n} \varphi_{\sigma^{k} \omega} \circ f_{\omega}^{k}-B_{\sigma_{n}^{2}(\omega)}^{\omega}=o\left(n^{\frac{1}{4}+\epsilon_{0}}\right) \text { a.s. }
$$

where $\epsilon_{0}$ is the same as the one in (2.2).

3. Coboundary: define $v:=d v_{\omega} d \mathbb{P}(\omega)$, then there is measurable function $g$ defined on $\left(\bigcup_{\omega \in \Omega}(\{\omega\} \times M), v\right)$ s.t.

$$
\varphi_{\sigma \omega} \circ f_{\omega}(x)=g_{\sigma \omega} \circ f_{\omega}(x)-g_{\omega}(x) \text { a.s. }-v .
$$

Moreover, if $\rho_{n}=e^{-a \cdot n^{b}}, g \in L^{\infty}(v)$; if $\rho_{n}=\frac{1}{n^{D}}, g \in L^{\frac{(D-2-\delta) \cdot(p-\gamma)}{(1+\delta) p}}(v)$ for sufficiently small $\delta$.

To prove Theorem 6.2, we need one probability lemma: 


\section{Lemma 6.3 (Transfer, see [Kal02] Theorem 6.10)}

Given probability spaces $(\Omega, \mathcal{F}, P),\left(\Omega^{\prime}, \mathcal{F}^{\prime}, P^{\prime}\right), T$ is Borel space, $S$ is measurable space. Random elements $\eta^{\prime}: \Omega^{\prime} \rightarrow T, \xi^{\prime}: \Omega^{\prime} \rightarrow S, \xi: \Omega \rightarrow S$ with $\xi \stackrel{d}{=} \xi^{\prime}$. Then there is measurable function $f: S \times[0,1] \rightarrow T$, if define random element $\eta:=f(\xi, U)$ with any uniform distribution on $[0,1]: U \sim U(0,1)$ independent of $\xi, f$, we have

$$
(\eta, \xi) \stackrel{d}{=}\left(\eta^{\prime}, \xi^{\prime}\right) \text {. }
$$

One way to have $\xi$ independent of $U$ is defining $\eta$ on product probability space $\left(\Omega \times[0,1], P \times \operatorname{Leb}_{[0,1]}\right)$, i.e. product extension of $(\Omega, P)$.

Proof [Proof of Theorem 6.2]

From Definition 6.1, there is RYT $(\Delta, F)$ such that $F_{\omega}^{R_{\omega}}=f_{\omega}^{R_{\omega}}$. Verify distortion (2.1) from (6.2): if separation time $s_{\omega}(x, y)=n$, then for any $i<n$, $F_{\omega}^{R_{\omega}^{i}(x)}(x), F_{\omega}^{R_{\omega}^{i}(y)}(y)$ lie in the same element of $\mathcal{P}_{\sigma_{\omega}^{R_{\omega}^{i}(x)}}$ and $F_{\omega}^{R_{\omega}^{n}(x)}(x), F_{\omega}^{R_{\omega}^{n}(y)}(y)$ lie in different elements of $\mathcal{P}_{\sigma_{\omega}^{R_{\omega}^{n}(x) \omega}}$. From (6.1), we have

$$
d(x, y) \leq \beta \cdot d\left(f_{\omega}^{R_{\omega}}(x), f_{\omega}^{R_{\omega}}(y)\right) \leq \cdots \leq \beta^{n} \cdot d\left(f_{\omega}^{R_{\omega}^{n}}(x), f_{\omega}^{R_{\omega}^{n}}(y)\right) \leq \beta^{n} \cdot \sup _{x, y \in M} d(x, y) .
$$

From (6.2), we have

$$
\left|\log \frac{J F_{\omega}^{R_{\omega}}(x)}{J F_{\omega}^{R_{\omega}}(y)}\right| \leq C \cdot d\left(f_{\omega}^{R_{\omega}}(x), f_{\omega}^{R_{\omega}}(y)\right) \leq C \cdot \sup _{x, y \in M} d(x, y) \cdot \beta^{n-1},
$$

that is, there is $C>0$ s.t.

$$
\left|\frac{J F_{\omega}^{R_{\omega}}(x)}{J F_{\omega}^{R_{\omega}}(y)}-1\right| \leq C \cdot \beta^{s_{\sigma} R_{\omega}(x)_{\omega}}\left(F_{\omega}^{R_{\omega}}(x), F_{\omega}^{R_{\omega}}(y)\right) \leq C \cdot\left(\beta^{\gamma}\right)^{S_{\sigma} R_{\omega}(x)_{\omega}}\left(F_{\omega}^{R_{\omega}}(x), F_{\omega}^{R_{\omega}}(y)\right) .
$$

Therefore, by Lemma 3.1, we construct a $\operatorname{RYT}(\Delta, F, \mu)$ satisfying all assumptions in Definition 2.1. Define projection $\pi_{\omega}: \Delta_{\omega} \rightarrow M$ by $\pi_{\omega}(x, l):=f_{\sigma^{-l} \omega}^{l}(x)$. It is semi-conjugacy, $v_{\omega}:=\left(\pi_{\omega}\right)_{*} \mu_{\omega}$ is equivariant probability measures, see section 3.1 in [BBR19]. $\phi_{\omega}:=\varphi_{\omega} \circ \pi_{\omega}$ is bounded above by $\max _{x \in M}|\varphi(x)|$ and fiberwise mean zero on $\left(\Delta_{\omega}, \mu_{\omega}\right)$. We claim

$$
\phi(\omega, \cdot):=\phi_{\omega}(\cdot) \in \mathcal{F}_{\beta^{\gamma}, \frac{p}{\gamma}}^{\mathcal{K}^{\gamma}} .
$$

For any $(x, l),(y, l) \in \Delta_{\omega}$ with $s_{\omega}((x, l),(y, l))=n$, by $(6.3)$,

$$
\begin{gathered}
\left|\phi_{\omega}(x, l)-\phi_{\omega}(y, l)\right|=\left|\varphi_{\omega}\left(f_{\sigma^{-l} \omega}^{l} x\right)-\varphi_{\omega}\left(f_{\sigma^{-l} \omega}^{l} y\right)\right| \leq C_{\varphi} \cdot d\left(f_{\sigma^{-l} \omega}^{l} x, f_{\sigma^{-l} \omega}^{l} y\right)^{\gamma} \\
\leq C_{\varphi} \cdot C^{\gamma} \cdot \mathcal{K}_{\omega}^{\gamma} \cdot d\left(f_{\sigma^{-l} \omega}^{R_{\sigma} l_{\omega}}(x), f_{\sigma^{-l} \omega}^{R_{-l} l_{\omega}}(y)\right)^{\gamma} \leq C_{\varphi} \cdot C^{\gamma} \cdot \mathcal{K}_{\omega}^{\gamma} \cdot\left(\beta^{\gamma}\right)^{n-1} \cdot \sup _{x, y \in M} d(x, y)^{\gamma}
\end{gathered}
$$




$$
\leq C_{\varphi} \cdot C^{\gamma} \cdot \mathcal{K}_{\omega}^{\gamma} \cdot \sup _{x, y \in M} d(x, y)^{\gamma} \cdot\left(\beta^{\gamma}\right)^{-1} \cdot\left(\beta^{\gamma}\right)^{s_{\omega}((x, l),(y, l))}
$$

So $\phi \in \mathcal{F}_{\beta^{\gamma}, \frac{p}{\gamma}}^{\mathcal{K}^{\gamma}}$ with Lipschitz constant $C_{\varphi} \cdot C^{\gamma} \cdot \sup _{x, y \in M} d(x, y)^{\gamma} \cdot\left(\beta^{\gamma}\right)^{-1}$. Apply Theorem 2.6, we have: there is $\sigma^{2} \geq 0$ s.t.

$$
\lim _{n \rightarrow \infty} \frac{\int\left(\sum_{k \leq n} \varphi_{\sigma^{k} \omega} \circ f_{\omega}^{k}\right)^{2} d v_{\omega}}{n}=\lim _{n \rightarrow \infty} \frac{\int\left(\sum_{k \leq n} \phi_{\sigma^{k}} \circ F_{\omega}^{k}\right)^{2} d \mu_{\omega}}{n}=\sigma^{2} .
$$

This proves the first statement of Theorem 6.2. It remains to show how the QASIP or Coboundary of this RYT are projected to the RDS: let

$$
\tau(\omega, x):=\left(\sigma \omega, F_{\omega}(x)\right),
$$

its transfer operator with respect to $\mu$ is $\tau^{*}$.

$$
\begin{gathered}
v:=d v_{\omega} d \mathbb{P}(\omega), \\
\chi(\omega, x):=\left(\sigma \omega, f_{\omega}(x)\right),
\end{gathered}
$$

its transfer operator with respect to $v$ is $\chi^{*}$. Define:

$$
\varphi^{\prime}(\omega, \cdot):=\varphi_{\omega}(\cdot)
$$

For Coboundary, if we have the Coboundary on $(\Delta, \mu)$ :

$$
\phi \circ \tau=g^{\prime} \circ \tau-g^{\prime} \text { a.s. }-\mu
$$

where $g^{\prime} \in L^{\frac{(D-2-\delta) \cdot(p-\gamma)}{(1+\delta) p}}(\Delta, \mu)$ (set $D=\infty$ if $\rho_{n}=e^{-a \cdot n^{b}}$ ), we want to show the Coboundary on $\left(\bigcup_{\omega \in \Omega}(\{\omega\} \times M), v\right)$ : there is measurable function $g$ on $\left(\bigcup_{\omega \in \Omega}(\{\omega\} \times\right.$ $M), v)$ s.t.

$$
\varphi^{\prime} \circ \chi=g \circ \chi-g \text { a.s. }-v,
$$

where $g \in L^{\frac{(D-2-\delta) \cdot(p-\gamma)}{(1+\delta) p}}\left(\bigcup_{\omega \in \Omega}(\{\omega\} \times M), v\right)$.

To do this, we will verify the conditions of Theorem 1.1 in [Liv96]:

Firstly, by ergodic theorem, a standard calculation gives

$$
\lim _{n \rightarrow \infty} \frac{\int\left(\sum_{i \leq n} \phi \circ \tau^{i}\right)^{2} d \mu}{n}=\int \phi^{2} d \mu+2 \sum_{i \geq 1} \int \phi \cdot \phi \circ \tau^{i} d \mu
$$

while

$$
\lim _{n \rightarrow \infty} \frac{\int\left(\sum_{i \leq n} \phi \circ \tau^{i}\right)^{2} d \mu}{n}=\lim _{n \rightarrow \infty} \frac{\int\left(g^{\prime} \circ \tau^{n+1}-g^{\prime}\right)^{2} d \mu}{n} \leq \lim _{n \rightarrow \infty} \frac{2 \int g^{\prime 2} d \mu}{n} \rightarrow 0,
$$


then

$$
\int \phi^{2} d \mu+2 \sum_{i \geq 1} \int \phi \cdot \phi \circ \tau^{i} d \mu=0
$$

Since $\phi(\omega, \cdot)=\varphi^{\prime}\left(\omega, \pi_{\omega}(\cdot)\right)$, so

$$
\int \varphi^{\prime 2} d v+2 \sum_{i \geq 1} \int \varphi^{\prime} \cdot \varphi^{\prime} \circ \chi^{i} d v=0
$$

Secondly, by Lemma 3.5 , there is $C=C_{\phi, h, F, \beta^{\gamma}, \delta, \frac{p}{\gamma}} \cdot\left\|\mathcal{K}^{\gamma}\right\|_{L^{\frac{p}{\gamma}}}$ s.t.

$$
\begin{gathered}
\sum_{i \geq 1}\left|\int \varphi^{\prime} \cdot \varphi^{\prime} \circ \chi^{i} d v\right|=\sum_{i \geq 1}\left|\int \phi \cdot \phi \circ \tau^{i} d \mu\right|=\sum_{i \geq 1}\left|\iint \phi_{\omega} \cdot \phi_{\sigma^{i} \omega} \circ F_{\omega}^{i} d \mu_{\omega} d \mathbb{P}\right| \\
\leq C_{\phi} \cdot \sum_{i \geq 1} \iint\left|P_{\omega}^{i}\left(\phi_{\omega}\right)\right| d \mu_{\sigma^{i} \omega} d \mathbb{P} \leq C \cdot \sum_{i \geq 1} \frac{1}{i^{(D-2-\delta) \cdot \frac{p-\gamma}{p}}}<\infty .
\end{gathered}
$$

Thirdly, by Lemma 3.5 , there is $C=C_{\phi, h, F, \beta^{\gamma}, \delta, \frac{p}{\gamma}} \cdot\left\|\mathcal{K}^{\gamma}\right\|_{L^{\frac{p}{\gamma}}}$ s.t.

$$
\begin{gathered}
\sum_{n \geq 1} \int\left|\left(\chi^{*}\right)^{n} \varphi^{\prime}\right| d v=\sum_{n \geq 1} \sup _{\|\psi\|_{L^{\infty} \leq 1}} \int \psi \circ \chi^{n} \cdot \varphi^{\prime} d v \\
=\sum_{n \geq 1} \sup _{\|\psi\|_{L^{\infty} \leq 1}} \iint \psi_{\sigma^{n} \omega} \circ \pi_{\omega} \circ F_{\omega}^{n} \cdot \phi_{\omega} d \mu_{\omega} d \mathbb{P} \\
\leq \sum_{n \geq 1} \iint\left|P_{\omega}^{n}\left(\phi_{\omega}\right)\right| d \mu_{\sigma^{n}} d \mathbb{P} \leq C \cdot \sum_{i \geq 1} \frac{1}{i^{(D-2-\delta) \cdot \frac{p-\gamma}{p}}<\infty .}
\end{gathered}
$$

So by Theorem 1.1 in [Liv96], there is measurable function $g$ on $\left(\bigcup_{\omega \in \Omega}(\{\omega\} \times\right.$ $M), v)$ s.t.

$$
\varphi^{\prime} \circ \chi=g \circ \chi-g \text { a.s. }-v
$$

where $g:=\sum_{i \geq 0}\left(\chi^{*}\right)^{n} \varphi^{\prime} \in L^{\frac{(D-2-\delta) \cdot(p-\gamma)}{(1+\delta) p}}\left(\bigcup_{\omega \in \Omega}(\{\omega\} \times M), v\right)$, this is because,

$\|g\|_{L} \frac{(D-2-\delta) \cdot(p-\gamma)}{(1+\delta) p} \leq \sum_{n \geq 0}\left\|\left(\chi^{*}\right)^{n} \varphi^{\prime}\right\|_{L} \frac{(D-2-\delta) \cdot(p-\gamma)}{(1+\delta) p} \leq C_{\varphi}+\sum_{n \geq 1}\left(\int\left|\left(\chi^{*}\right)^{n} \varphi^{\prime}\right| d v\right)^{\frac{(1+\delta) p}{(D-2-\delta) \cdot(p-\gamma)}}$

using the same estimate of (6.4), the above inquality becomes

$$
\leq C_{\varphi}+C \cdot \sum_{n \geq 1} \frac{1}{n^{(D-2-\delta) \cdot \frac{p-\gamma}{p} \cdot \frac{(1+\delta) p}{(D-2-\delta) \cdot(p-\gamma)}}} \leq C_{\varphi}+C \cdot \sum_{n \geq 1} \frac{1}{n^{1+\delta}}<\infty .
$$


So we finish the proof of Coboundary.

For QASIP, by (2.2), we have Brownian motion $\bar{B}^{\omega}$ defined on an extended probability space $\left(\boldsymbol{\Delta}_{\omega}, \mathbb{Q}_{\omega}\right)$ and

$$
\begin{gathered}
\sum_{k \leq n} \varphi_{\sigma^{k} \omega} \circ f_{\omega}^{k} \circ \pi_{\omega}-\bar{B}_{\sigma_{n}^{2}(\omega)}^{\omega}=\sum_{k \leq n} \phi_{\sigma^{k} \omega} \circ F_{\omega}^{k}-\bar{B}_{\sigma_{n}^{2}(\omega)}^{\omega}=O\left(n^{\frac{1}{4}+\epsilon_{0}}\right) \text { a.s. }-\mathbb{Q}_{\omega}, \\
\sigma_{n}^{2}(\omega)=\int\left(\sum_{k \leq n} \varphi_{\sigma^{k} \omega} \circ f_{\omega}^{k} \circ \pi_{\omega}\right)^{2} d \mu_{\omega}=\int\left(\sum_{k \leq n} \varphi_{\sigma^{k} \omega} \circ f_{\omega}^{k}\right)^{2} d v_{\omega} .
\end{gathered}
$$

By Lemma 6.3, there is a function $H: \mathbb{R}^{\mathbb{N}} \times[0,1] \rightarrow \mathbb{R}^{\mathbb{N}}$ and a Brownian motion $\hat{B}^{\omega}$ such that

$$
\begin{gathered}
\mathbb{Q}_{\omega}\left(\left(\left(\varphi_{\sigma^{k} \omega} \circ f_{\omega}^{k} \circ \pi_{\omega}\right)_{k \geq 1},\left(\bar{B}_{\sigma_{k}^{2}(\omega)}^{\omega}-\bar{B}_{\sigma_{k-1}^{2}(\omega)}^{\omega}\right)_{k \geq 1}\right) \in(\cdot, \cdot)\right)= \\
\left(\mu_{\omega} \times \operatorname{Leb}_{[0,1]}\right)\left(\left(\left(\varphi_{\sigma^{k} \omega} \circ f_{\omega}^{k} \circ \pi_{\omega}\right)_{k \geq 1},\left(\hat{B}_{\sigma_{k}^{2}(\omega)}^{\omega}-\hat{B}_{\sigma_{k-1}^{2}}^{\omega}(\omega)\right)_{k \geq 1}\right) \in(\cdot, \cdot)\right), \\
\sum_{k \leq n} \varphi_{\sigma^{k} \omega} \circ f_{\omega}^{k} \circ \pi_{\omega}-\hat{B}_{\sigma_{n}^{2}(\omega)}^{\omega}=O\left(n^{\frac{1}{4}+\epsilon_{0}}\right) \text { a.s.- }\left(\mu_{\omega} \times \operatorname{Leb}_{[0,1]}\right),
\end{gathered}
$$

where $\left(\hat{B}_{\sigma_{k}^{2}(\omega)}^{\omega}-\hat{B}_{\sigma_{k-1}^{2}(\omega)}^{\omega}\right)_{k \geq 1}=H\left(\left(\varphi_{\sigma^{k} \omega} \circ f_{\omega}^{k} \circ \pi_{\omega}\right)_{k \geq 1}, U\right)$ forms a Brownian motion defined on $\left(\Delta_{\omega} \times[0,1], \mu_{\omega} \times \operatorname{Leb}_{[0,1]}\right)$. Then $\left(B_{\sigma_{k}^{2}(\omega)}^{\omega}-B_{\sigma_{k-1}^{2}(\omega)}^{\omega}\right)_{k \geq 1}:=H\left(\left(\varphi_{\sigma^{k} \omega} \circ\right.\right.$ $\left.\left.f_{\omega}^{k}\right)_{k \geq 1}, U\right)$ also forms a Brownian motion defined on $\left(M \times[0,1], v_{\omega} \times \operatorname{Leb}_{[0,1]}\right)$. Hence

$$
\sum_{k \leq n} \varphi_{\sigma^{k} \omega} \circ f_{\omega}^{k}-B_{\sigma_{n}^{2}(\omega)}^{\omega}=O\left(n^{\frac{1}{4}+\epsilon_{0}}\right) \text { a.s. }-\left(v_{\omega} \times \operatorname{Leb}_{[0,1]}\right) .
$$

Here $\mathbf{M}_{\omega}:=\left(M \times[0,1], v_{\omega} \times \operatorname{Leb}_{[0,1]}\right)$.

\section{Applications}

We will apply Theorem 6.2 to the following RDS via verifying the six conditions (1)-(6) in Definition 6.1: i.i.d. translations of unimodal maps (satisfying ColletEckmann condition) in [BBMD02], non-uniformly expanding maps (with slow recurrence to singularities) in [AA03] or [AV13], i.i.d. perturbations of admissible S-unimodal maps (satisfying Collet-Eckmann condition or summability condition of exponent 1) in [Du15] and LSV maps possessing an indifferent fixed point in [BBR19]. i.i.d. here means $f_{\sigma^{i} \omega}$ only depends on $\omega_{i}$, then for any $n \in \mathbb{N}, f_{\sigma^{n} \omega}$ is independent of $\left(f_{\sigma^{i} \omega}\right)_{i \leq n-1}$. In Definition 6.1, conditions (1), (2), (4), (6.1) and (6.2) are naturally satisfied when random Young tower is constructed. Condition (3) is also satisfied since $\left\{R_{\omega}=n\right\}$ is constructed inductively, so only depends on $\left(f_{\sigma^{i} \omega}\right)_{0 \leq i \leq n-1}$, i.e. $\omega_{0}, \omega_{1}, \cdots, \omega_{n-1}$. So it remains to verify conditions (6) and (6.3): 


\section{i.i.d. translations of unimodal maps, see [BBMD02]}

Conditions (6.3) holds due to Lemma 7.9, 7.10, 9.1. Condition (6) is due to Proposition 8.3 in [BBMD02].

\section{i.i.d. perturbations of S-unimodal maps, see [Du15]}

Condition (6) is due to Theorem 8.1.2, 8.1.4 in [Du15].

- For S-unimodal maps satisfying Collet-Eckmann condition in [Du15], condition (6.3) is due to Proposition 8.3.5 in [Du15].

- For S-unimodal maps on interval $I$ satisfying summability condition of exponent 1 in [Du15], we will verify condition (6.3) with $\mathcal{K} . \in L^{\infty}(\Omega)$ :

$$
d\left(f_{\omega}^{k}(x), f_{\omega}^{k}(y)\right) \leq C \cdot d\left(f_{\omega}^{R_{\omega}}(x), f_{\omega}^{R_{\omega}}(y)\right)
$$

where $k \leq R_{\omega}=n, x, y \in U_{\omega}(z, n):=\left(f_{\omega}^{n}\right)^{-1}(\widetilde{B}(\delta)) \bigcap J_{z, n}^{\omega}, n$ is a $\theta$-good return time of $(\omega, z)$ into $\widetilde{B}(\delta),\left.f_{\omega}^{n}\right|_{U_{\omega}(z, n)}$ is diffeomorphism onto $\widetilde{B}(\delta)$. Equivalently, we will show

$$
\left|D f_{\sigma^{k} \omega}^{n-k}\right| f_{\omega}^{n}\left(U_{\omega}(z, n)\right) \mid \geq \frac{1}{C}
$$

where $n-k$ is a $\theta$-good return time of $\left(\sigma^{k} \omega, f_{\omega}^{k}(z)\right)$ into $\widetilde{B}(\delta), f_{\sigma^{k} \omega}^{n-k}$ is diffeomorphism from $f_{\omega}^{n}\left(U_{\omega}(z, n)\right) \subseteq U_{\sigma^{k}}\left(f_{\omega}^{k}(z), n-k\right)$ onto $\widetilde{B}(\delta)$ (see Lemma 8.2.1, Proposition 8.2.3 and Proposition 8.2.4 in [Du15]). By Lemma 8.2.1 in [Du15] distortion: for any $z_{1}, z_{2} \in U_{\sigma^{k} \omega}\left(f_{\omega}^{k}(z), n-k\right)$,

$$
e^{-\frac{1}{2}} \leq \frac{\left|D f_{\sigma^{k} \omega}^{n-k}\left(z_{1}\right)\right|}{\left|D f_{\sigma^{k} \omega}^{n-k}\left(z_{2}\right)\right|} \leq e^{\frac{1}{2}}
$$

Then for any $z_{1} \in f_{\omega}^{n}\left(U_{\omega}(z, n)\right)$,

$$
\left|D f_{\sigma^{k} \omega}^{n-k}\left(z_{1}\right)\right| \geq e^{-\frac{1}{2}} \cdot \frac{\left|f_{\sigma^{k} \omega}^{n-k}\left(U_{\sigma^{k}}\left(f_{\omega}^{k}(z), n-k\right)\right)\right|}{\left|U_{\sigma^{k} \omega}\left(f_{\omega}^{k}(z), n-k\right)\right|}=\frac{|\widetilde{B}(\delta)|}{|I|}:=\frac{1}{C},
$$

so (6.3) holds.

\section{i.i.d. translations of non-uniformly expanding maps, see [AV13]}

Condition (6) is due to Proposition 5.1, Section 5.2.2 and Theorem 2.9 in [AV13]. Condition (6.3) is due to Proposition 4.9 in [AV13].

\section{i.i.d. perturbations of LSV maps with neutral fixed point, see [BBR19]}

(6.3) is because LSV maps have derivative no less than 1 , so $d\left(f_{\omega}^{k}(x), f_{\omega}^{k}(y)\right) \leq$ $d\left(f_{\omega}^{R_{\omega}}(x), f_{\omega}^{R_{\omega}}(y)\right)$. Condition (6) is due to (5.5) and Proposition 5.3 in [BBR19] with $\frac{1}{\alpha_{0}}>6$, that is, QASIP holds for $\Omega=\left[\alpha_{0}, \alpha_{1}\right]^{\mathbb{Z}}$ where $0<\alpha_{0}<\frac{1}{6}, \alpha_{1}<1$. 


\section{Acknowledgments}

The author warmly thanks his advisor Prof. Andrew Török for posing him the question in this paper, and the support during the author studies at University of Houston. The author also thanks University of Houston for good place to study dynamical system.

\section{References}

[AA03] José Ferreira Alves and Vítor Araújo, Random perturbations of nonuniformly expanding maps, Astérisque (2003), no. 286, xvii, 25-62, Geometric methods in dynamics. I. MR 2052296

[AA16] Mohamed Abdelkader and Romain Aimino, On the quenched central limit theorem for random dynamical systems, J. Phys. A 49 (2016), no. 24, 244002, 13. MR 3512078

[ALS09] Arvind Ayyer, Carlangelo Liverani, and Mikko Stenlund, Quenched CLT for random toral automorphism, Discrete Contin. Dyn. Syst. 24 (2009), no. 2, 331-348. MR 2486579

[AV13] José F. Alves and Helder Vilarinho, Strong stochastic stability for nonuniformly expanding maps, Ergodic Theory Dynam. Systems 33 (2013), no. 3, 647-692. MR 3062897

[BBMD02] Viviane Baladi, Michael Benedicks, and Véronique Maume-Deschamps, Almost sure rates of mixing for i.i.d. unimodal maps, Ann. Sci. École Norm. Sup. (4) 35 (2002), no. 1, 77-126. MR 1886006

[BBR19] Wael Bahsoun, Christopher Bose, and Marks Ruziboev, Quenched decay of correlations for slowly mixing systems, Trans. Amer. Math. Soc. (2019).

[DFGTV18] D. Dragičević, G. Froyland, C. González-Tokman, and S. Vaienti, A spectral approach for quenched limit theorems for random expanding dynamical systems, Comm. Math. Phys. 360 (2018), no. 3, 1121-1187. MR 3803820

[Du15] Zhikun Du, On mixing rates for random perturbations, ProQuest LLC, Ann Arbor, MI, 2015, Thesis (Ph.D.)-National University of Singapore (Singapore). MR 3474622 
[HL18] Olli Hella and Juho Leppänen, Central limit theorems with a rate of convergence for time-dependent intermittent maps, arXiv e-prints (2018), arXiv:1811.11170.

[HS18] Olli Hella and Mikko Stenlund, Quenched normal approximation for random sequences of transformations, arXiv e-prints (2018), arXiv:1810.10760.

[Kal02] Olav Kallenberg, Foundations of modern probability, second ed., Probability and its Applications (New York), Springer-Verlag, New York, 2002. MR 1876169

[Kif98] Yuri Kifer, Limit theorems for random transformations and processes in random environments, Trans. Amer. Math. Soc. 350 (1998), no. 4, 1481-1518. MR 1451607

[Liv96] Carlangelo Liverani, Central limit theorem for deterministic systems, International Conference on Dynamical Systems (Montevideo, 1995), Pitman Res. Notes Math. Ser., vol. 362, Longman, Harlow, 1996, pp. 56-75. MR 1460797

[LV18] Xin Li and Helder Vilarinho, Almost sure mixing rates for nonuniformly expanding maps, Stoch. Dyn. 18 (2018), no. 4, 1850027, 34. MR 3842250

[SH83] D. J. Scott and R. M. Huggins, On the embedding of processes in Brownian motion and the law of the iterated logarithm for reverse martingales, Bull. Austral. Math. Soc. 27 (1983), no. 3, 443-459. MR 715321

[Su19a] Yaofeng Su, Almost Surely Invariance Principle for Non-stationary and Random Intermittent Dynamical Systems, to appear in Discrete Contin. Dyn. Syst. (A) (2019), arXiv:1903.09758.

[Su19b] _ Vector-valued Almost Sure Invariance Principle For Non-stationary Dynamical Systems, arXiv e-prints (2019), arXiv:1903.09763. 